\title{
Heterodyne Receiver for Origins
}

Martina C. Wiedner $\odot,{ }^{\mathrm{a} *}$ Susanne Aalto, ${ }^{\mathrm{b}}$ Edward G. Amatucci $\odot,{ }^{\mathrm{c}}$
Andrey Baryshev, ${ }^{\mathrm{d}}$ Cara Battersby $\odot$, ${ }^{\mathrm{e}}$ Victor Belitsky, ${ }^{\mathrm{f}}$ Edwin A. Bergin,
Bruno Borgo, ${ }^{\mathrm{h}}$ Ruth C. Carter, ${ }^{\mathrm{c}}$ Emmanuel Caux ${ }^{\text {i }}{ }^{\mathrm{i}}$ Asantha Cooray, James A. Corsetti, ${ }^{\text {c Elvire De Beck } \odot,{ }^{b} \text { Yan Delorme, }{ }^{\text {a }} \text { Vincent Desmaris, }}$ Michael J. DiPirro, ${ }^{\text {c }}$ Brian Ellison ${ }^{\text {k }}$ Anna M. Di Giorgio, Martin Eggens $\odot,{ }^{\text {m }}$ Juan-Daniel Gallego $\odot,{ }^{\text {n }}$ Maryvonne Gerin $\odot,{ }^{\text {a }}$ Paul F. Goldsmith $\odot,{ }^{o}$ Christophe Goldstein, ${ }^{\text {p }}$ Frank Helmich $\odot,{ }^{\text {m,q }}$ Fabrice Herpin $\odot{ }^{\mathrm{r}}$ Richard E. Hills $\odot{ }^{\mathrm{s}}$ Michiel R. Hogerheijde $\odot$, Leslie K. Hunt $\odot$, ${ }^{\text {v }}$ Willem Jellema $\odot{ }^{\text {m }}$ Geert Keizer, ${ }^{\text {m }}$ Jean-Michel Krieg, Gabby Kroes, ${ }^{\text {w }}$ Philippe Laporte, ${ }^{\mathrm{x}}$ André Laurens, ${ }^{\mathrm{p}}$ David T. Leisawitz $₫,{ }^{\mathrm{c}}$

Dariusz C. Lis $\odot,{ }^{\text {,o }}$ Gregory E. Martins, ${ }^{\text {c Imran Mehdi, }}$ Margaret Meixner, ${ }^{c, y, z}$ Gary Melnick, ${ }^{\text {aa }}$ Stefanie N. Milam $\odot$, David A. Neufeld, ${ }^{\mathrm{z}}$ Napoléon Nguyen Tuong, ${ }^{\mathrm{h}}$ René Plume, ${ }^{\text {ab }}$ Klaus M. Pontoppidan $\odot{ }^{\mathrm{y}}$ Benjamin Quertier-Dagorn, ${ }^{\mathrm{r}}$ Christophe Risacher $\odot$, ${ }^{\text {ac,ad }}$ Johannes G. Staguhn $\odot,{ }^{\text {c,z }}$ Edward Tong, ${ }^{\text {aa }}$ Serena Viti, ${ }^{\text {ae }}$ Friedrich Wyrowski, ${ }^{\text {ac }}$ and

The Origins Space Telescope Mission Concept Study Team ${ }^{\dagger}$

a'Laboratoire d'Etudes du Rayonnement et de la Matière en Astrophysique et Atmosphères (LERMA), Observatoire de Paris, Université Paris Sciences \& Lettres (PSL) Centre National de la Recherche Scientifique (CNRS), Sorbonne Université, Paris, France

${ }^{\mathrm{b}}$ Chalmers University of Technology, Department of Space, Earth and Environment, Onsala Space Observatory, Onsala, Sweden

${ }^{c}$ NASA Goddard Space Flight Center, Greenbelt, Maryland, United States ${ }^{\mathrm{d}}$ University of Groningen, Kapteyn Astronomical Institute, Groningen, The Netherlands

${ }^{\mathrm{e}}$ University of Connecticut, Department of Physics, Storrs, Connecticut, United States

${ }^{\mathrm{f}}$ Chalmers University of Technology, Group for Advanced Receiver Development, Department of Space, Earth and Environment, Gothenburg, Sweden

${ }^{g}$ University of Michigan, Department of Astronomy, Ann Arbor, Michigan, United States

${ }^{h}$ Laboratoire d'Etudes Spatiales et d'Instrumentation en Astrophysique (LESIA), Observatoire de Paris, Université Paris Sciences \& Lettres, Centre National de la Recherche Scientifique, Sorbonne University, University Paris Diderot, Paris, France institut de Recherche en Astrophysique et Planétologie, Université de Toulouse, Centre National de la Recherche Scientifique, Centre national d'études spatiales, Université Toulouse III - Paul Sabatier, Toulouse, France

${ }^{j}$ University of California, Center for Cosmology, Department of Physics and Astronomy, Irvine, California, United States

${ }^{\mathrm{k}}$ Science and Technology Facilities Council Rutherford Appleton Laboratory, Didcot, United Kingdom

${ }^{1}$ Istituto Nazionale di Astrofisica-Istituto di Astrofisica e Planetologia Spaziali, Roma, Italy

${ }^{\mathrm{m}}$ SRON Netherlands Institute for Space Research, Groningen, The Netherlands

${ }^{\mathrm{n}}$ Centro Astronómico de Yebes, Centro Astronómico de Yebes, Observatorio Astronómico Nacional, Guadalajara, Spain

${ }^{\circ}$ Jet Propulsion Laboratory, California Institute of Technology, Pasadena, California, United States

${ }^{\mathrm{P}}$ Centre National d'Études Spatiales, Toulouse, France

${ }^{\mathrm{q}}$ University of Groningen, Kapteyn Institute, Groningen, The Netherlands 'Laboratoire d'Astrophysique de Bordeaux, Université de Bordeaux, Centre National de la Recherche Scientifique, Pessac, France 
${ }^{\mathrm{s}}$ University of Cambridge, Astrophysics Group, Cavendish Laboratory, Cambridge, United Kingdom

'Leiden University, Leiden Observatory, Leiden, The Netherlands

"University of Amsterdam, Anton Pannekoek Institute for Astronomy, Amsterdam, The Netherlands

${ }^{\vee}$ Istituto Nazionale di Astrofisica-Osservatorio Astrofisico di Arcetri, Firenze, Italy

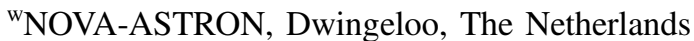

${ }^{\mathrm{x}}$ GEPI, Observatoire de Paris, Centre National de la Recherche Scientifique, Paris, France

${ }^{y}$ Space Telescope Science Institute, Baltimore, Maryland, United States

${ }^{\mathrm{z}}$ The Johns Hopkins University, Department of Physics and Astronomy, Baltimore, Maryland, United States

${ }^{a}$ Harvard-Smithsonian Center for Astrophysics, Cambridge, Massachusetts, United States

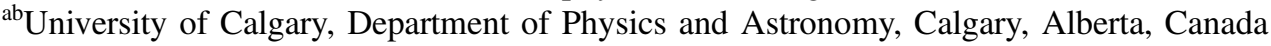

${ }^{\mathrm{ac}}$ Max-Planck-Institut für Radioastronomie, Bonn, Germany

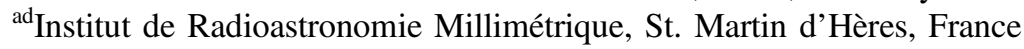

${ }^{\text {ae } U n i v e r s i t y ~ C o l l e g e ~ L o n d o n, ~ D e p a r t m e n t ~ o f ~ P h y s i c s ~ a n d ~ A s t r o n o m y, ~ L o n d o n, ~}$ United Kingdom

Abstract. The Heterodyne Receiver for Origins (HERO) is the first detailed study of a heterodyne focal plane array receiver for space applications. HERO gives the Origins Space Telescope the capability to observe at very high spectral resolution $\left(R=10^{7}\right)$ over an unprecedentedly large far-infrared (FIR) wavelengths range (111 to $617 \mu \mathrm{m})$ with high sensitivity, with simultaneous dual polarization and dual-frequency band operation. The design is based on prior successful heterodyne receivers, such as Heterodyne Instrument for the Far-Infrared /Herschel, but surpasses it by one to two orders of magnitude by exploiting the latest technological developments. Innovative components are used to keep the required satellite resources low and thus allowing for the first time a convincing design of a large format heterodyne array receiver for space. HERO on Origins is a unique tool to explore the FIR universe and extends the enormous potential of submillimeter astronomical spectroscopy into new areas of astronomical research. () The Authors. Published by SPIE under a Creative Commons Attribution 4.0 Unported License. Distribution or reproduction of this work in whole or in part requires full attribution of the original publication, including its DOI. [DOI: 10.1117/1.JATIS.7.1.011007]

Keywords: Origins; far-infrared; submillimeter; terahertz; heterodyne receiver; instrument design; space mission.

Paper 20081SS received Jun. 15, 2020; accepted for publication Dec. 9, 2020; published online Jan. 20, 2021.

\section{Introduction}

The Origins Space Telescope (Origins) ${ }^{1-4}$ traces our cosmic history, from the formation of the first galaxies and the rise of metals to the development of habitable worlds and present-day life. Origins does this through exquisite sensitivity to infrared radiation from ions, atoms, dust, and molecules especially water in the form of vapor and ice. Origins observes extra-solar planetary atmospheres, protoplanetary disks, and large-area extragalactic fields in the wavelength range of 2.8 to $588 \mu \mathrm{m}$ and is more than 1000 times more sensitive than its predecessors due to its large, cold $(4.5 \mathrm{~K})$ telescope and advanced instruments.

Origins has three baseline instruments- the Origins Survey Spectrometer (OSS), ${ }^{5}$ a farinfrared (FIR) bolometer array with a grating, Fourier transform spectrometer, and a FabryPérot; the Far-infrared Imager Polarimeter (FIP) ${ }^{6}$ with two large FIR bolometer arrays at 50 and $250 \mu \mathrm{m}$, and the Mid-Infrared Spectrometer Camera Transit spectrometer (MISC-T), ${ }^{7}$

\footnotetext{
*Address all correspondence to Martina C. Wiedner, martina.wiedner@obspm.fr

${ }^{\dagger}$ Members of the study team are listed in the Origins Space Telescope Mission Concept Study Report, which is available at https://asd.gsfc .nasa.gov/firs/docs/.
} 
Table 1 Summary of the performance of HERO. HERO can carry out dual polarization and dualfrequency observations over a large frequency range, i.e., effectively using $2 \times 2 \times 9$ pixels at the same time. The receiver is ideally suited for very high spectral resolution observations of many molecular and atomic lines that are critical for answering the key science questions discussed above.

\begin{tabular}{|c|c|c|c|c|c|c|c|c|}
\hline Band & $\begin{array}{l}\text { Frequency } \\
\quad(\mathrm{GHz})\end{array}$ & $\begin{array}{l}\text { Wavelength } \\
(\mu \mathrm{m})\end{array}$ & Pixels & $\begin{array}{c}T_{r x} \\
\text { DSB } \\
(\mathrm{K})\end{array}$ & $\begin{array}{c}\text { Sensitivity } \\
(\mathrm{mK}) \text { in } \\
1 \mathrm{~h} \text { at } \\
R=10^{6} \\
1 \sigma\end{array}$ & $\begin{array}{c}\text { Sensitivity } \\
(\mathrm{W}) \text { in } \\
1 \mathrm{~h} \text { at } \\
R=10^{6} \\
1 \sigma\end{array}$ & $\begin{array}{c}\text { Sensitivity } \\
\left(\mathrm{W} \mathrm{m}^{-2}\right) \\
\text { in } 1 \mathrm{~h} \\
5 \sigma\end{array}$ & $\begin{array}{l}\text { Examples of } \\
\text { spectral lines }\end{array}$ \\
\hline 1 & $486-756$ & 617-397 & $2 \times 9$ & 50 & 2.6 & $2.3 * 10^{-20}$ & $4.5 * 10^{-21}$ & $\begin{array}{l}\mathrm{H}_{2} \mathrm{O}, \mathrm{H}_{2}^{18} \mathrm{O}, \mathrm{HDO} \text {, } \\
\text { and } \mathrm{NH}_{3}\end{array}$ \\
\hline 2 & $756-1188$ & $397-252$ & $2 \times 9$ & 100 & 4.2 & $5.7 * 10^{-20}$ & $1.1 * 10^{-20}$ & $\begin{array}{l}\mathrm{H}_{2} \mathrm{O}, \mathrm{H}_{2}^{18} \mathrm{O} \text {, and } \\
\mathrm{H}_{3} \mathrm{O}^{+}\end{array}$ \\
\hline 3 & 1188-1782 & $252-168$ & $2 \times 9$ & 200 & 6.8 & $1.4 * 10^{-19}$ & $2.8 * 10^{-20}$ & $\begin{array}{l}\mathrm{H}_{2} \mathrm{O}, \mathrm{H}_{2}^{18} \mathrm{O}, \mathrm{NH}_{3} \text {, } \\
\text { and [NII] }\end{array}$ \\
\hline 4 & $1782-2700$ & $168-111$ & $2 \times 9$ & 300 & 8.4 & $2.6 * 10^{-19}$ & $5.2 * 10^{-20}$ & $\begin{array}{l}\mathrm{HD},[\mathrm{OI}],[\mathrm{NII} \text {, } \\
\text { and [CII] }\end{array}$ \\
\hline
\end{tabular}

DBS = double side band, $T_{\mathrm{rx}}=$ receiver temperature.

an ultrastable mid-infrared (mid-IR) spectrometer. To fully exploit the capabilities of Origins, two upscope instruments have been designed: the Mid-Infrared Spectrometer and Camera: Wide Field Imager (MISC-WFI) ${ }^{7}$ and the Heterodyne Receiver for Origins (HERO).

HERO's principal goal is to trace the trail of water ${ }^{8-10}$ from the interstellar medium (ISM), through the different stages of star formation to (habitable) planets. HERO will measure the amount, location, and temperature of water and its isotopologues in the ISM, protostars, and protoplanatary disks. This science goal requires HERO to have extremely high spectral resolution of up to $10^{7}$, to cover the major water lines and their isotopologues between 500 and $2700 \mathrm{GHz}$, and to have moderate instantaneous fields of view of a few arc minutes, sufficient to observe water in starless cores and protostars. This led the HERO team to design the first heterodyne focal plane array (FPA) receiver for a space mission. Table 1 summarizes HERO's capabilities. Figure 1 shows a block diagram of HERO. HERO has two sets of four frequency bands, one set for each linear polarization. Each frequency band has a small $3 \times 3$ pixel FPA.

Section 2 describes the instruments and its components in some detail. Section 3 puts HERO in the context of the Origins mission: it describes the resources HERO requires, the risks it carries, and shows the performance the receiver achieves, including comparison with prior missions. Section 4 discusses the technology roadmap. Section 5 briefly mentions an extension of HERO to be used as an element of the Event Horizon Telescope. The paper concludes with a summary and an outlook of the future.

\section{Instrument Description}

HERO uses the standard principle of a heterodyne receiver, in which the signal from the sky is mixed with a much stronger internally produced monochromatic reference signal called the local oscillator (LO). The beat signal is at the difference frequency, called the intermediate frequency (IF), which is amplified and spectrally analyzed. This beating of the two signals downconverts the sky signal to a lower frequency for amplification and makes very high spectral resolution measurements straightforward (spectral resolution up to $10^{11}$ have been demonstrated ${ }^{11}$ ). Extremely low noise mixers and first stage IF amplifiers are required for high sensitivity of such receivers and the tuning range of the mixers along with that of the LO determines the bandwidth of the receiver.

The design builds, in particular, on the experience from the receivers of the Atacama Large Millimeter Array (ALMA), ${ }^{12}$ the German REceiver at THz (GREAT)/ 4GREAT/ up-GREAT ${ }^{13,14}$ on the Stratospheric Observatory For Infrared Astronomy (SOFIA), and the Heterodyne 


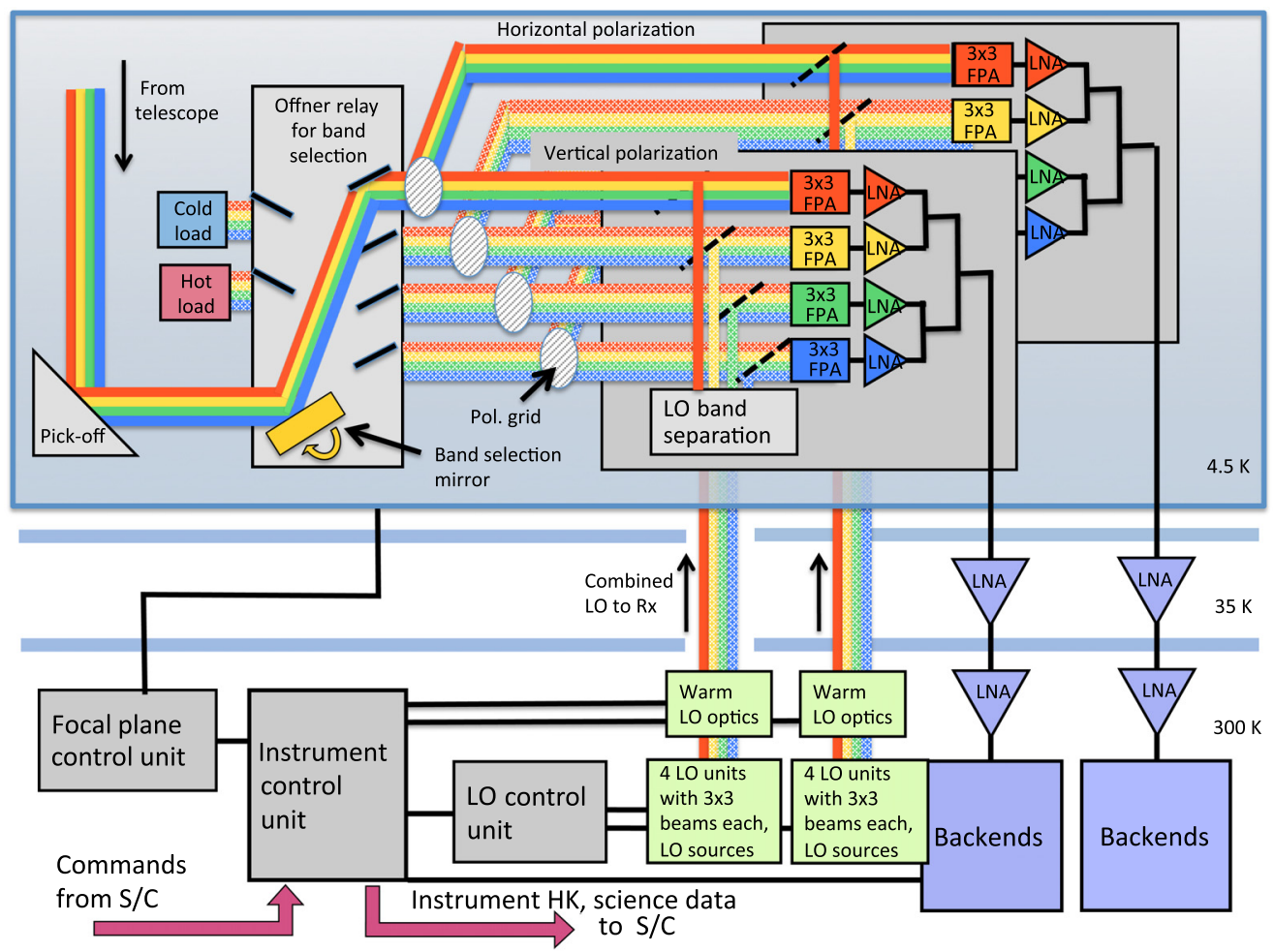

Fig. 1 Block diagram of HERO. The HERO instrument on Origins can observe and spectrally resolve the Universe over a wide submillimeter wavelength range in both linear polarizations simultaneously. HERO is a heterodyne receiver and has dual polarization $3 \times 3$ pixel focal plane heterodyne mixer arrays in four frequency bands.

Instrument for the Far-Infrared (HIFI) ${ }^{15}$ on the Herschel satellite. The HERO design also takes the experience from the first ground and airplane-based FPAs ${ }^{14,16-20}$ into account. HERO goes well beyond these earlier instruments by combining the largest frequency coverage of any heterodyne receiver and incorporates the first FPA design for space. The difficulty of cooling in space and the limited electrical power required us to look for innovative solutions for most of the receiver components. An earlier pape ${ }^{21}$ describes the available technologies and justifies the choices we made for a preliminary version of HERO, here we describe the design submitted to the Decadal Review and the remaining challenges that need to be addressed with a technology roadmap.

\subsection{Instrument Overview}

Figures 1 and 2 show the HERO receiver. HERO covers a frequency range of 486 to $2700 \mathrm{GHz}$ in only four frequency bands, in two linear polarizations. A band selection mirror in the Offner Relay directs the sky signal to two of the $3 \times 3$ mixer FPA, one for each polarization. The LO signals are generated in the spacecraft bus and are sent to the mixers. Low noise amplifiers (LNA) operating at $4.5 \mathrm{~K}$ and $35 \mathrm{~K}$ amplify the IF signal. In the warm spacecraft bus, the IF signal is amplified further and digitized, and the backends carry out spectral analysis via fast Fourier transforms (FFT). The LO, instrument, and focal plane control units control all aspects of the operation of the receiver.

\subsection{Optics}

\subsubsection{Design of optics}

Figure 3 shows the optical ray trace of HERO and Fig. 4 details of the Offner Relay, the cold receiver optics with the cold LO optics and the warm LO optics. The Origins Space Telescope 


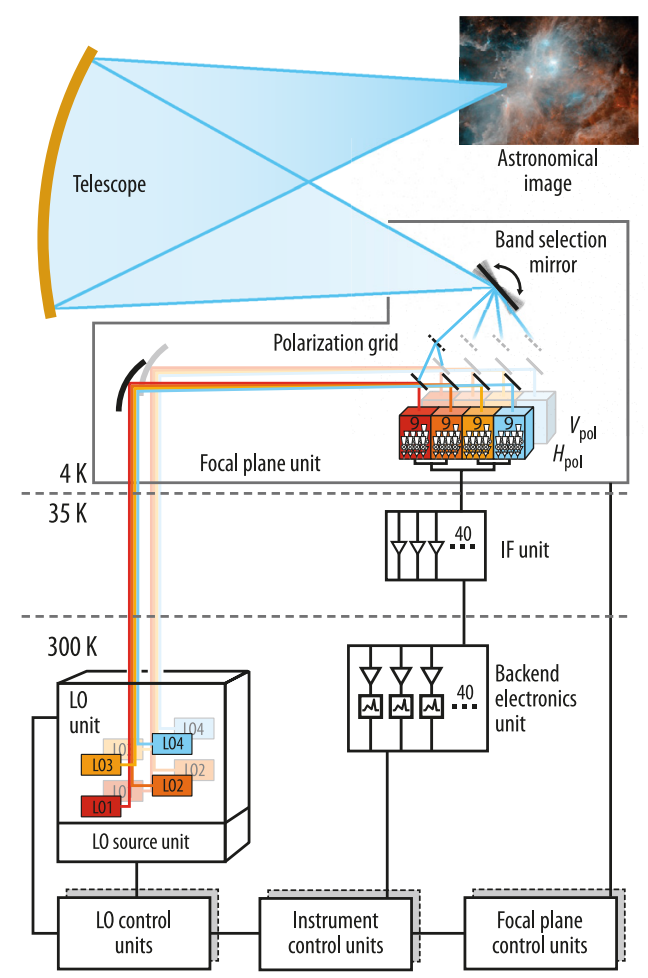

\begin{tabular}{|c|c|c|}
\hline Sub-system & Component & Hero \\
\hline \multirow{4}{*}{$\begin{array}{l}\text { Local } \\
\text { oscillator }\end{array}$} & $\begin{array}{l}\text { Synthesizer } \\
\text { technology }\end{array}$ & $\begin{array}{l}\text { CMOS or Y|G- } \\
\text { based W-band } \\
\text { synthesizer + } \\
\text { GaN. amps }\end{array}$ \\
\hline & Multiplied LO & $\begin{array}{c}\text { Cascaded multipl. } \\
+ \text { on-chip. power } \\
\text { combining }+a \\
\text { 3D integ. }\end{array}$ \\
\hline & DC power/pixel & $2 W$ \\
\hline & $\begin{array}{l}\text { Fractional } \\
\text { bandwidth }\end{array}$ & $45 \%$ \\
\hline \multirow{5}{*}{$\begin{array}{l}\text { Cryogenic } \\
\text { detectors }\end{array}$} & $\begin{array}{c}\text { Mixer } \\
\text { technology }\end{array}$ & SIS, HEB \\
\hline & $\begin{array}{l}\text { LNA } \\
\text { technology }\end{array}$ & $\begin{array}{l}\text { Low-power } \\
\text { SiGe HBT }\end{array}$ \\
\hline & Number of pixels & $\begin{array}{c}2 \text { (polarizations) } \\
\times 3 \times 3\end{array}$ \\
\hline & DC power/pixel & $0.5 \mathrm{~mW}$ \\
\hline & $\begin{array}{l}\text { Mixer. } \\
\text { assembly }\end{array}$ & Waveguide \\
\hline \multirow{4}{*}{ Backend } & IF processing & GaAs HEMT ampl \\
\hline & Spectrometer tech. & CMOS based SOC \\
\hline & DC power/pixel & $2 W$ \\
\hline & IF bandwidth & $8 \mathrm{GHz}$ \\
\hline \multicolumn{2}{|c|}{ Total DC power per pixel } & $3.5 \mathrm{~W}$ \\
\hline
\end{tabular}

Fig. 2 HERO is the first heterodyne array receiver designed for space. The schematic diagram shows a relatively simple design that provides the widest frequency coverage and the highest sensitivity of any heterodyne receiver. HERO uses sensitive state-of-the-art components with low power consumption and weight.

has a 5.9-m on-axis primary mirror. A secondary mirror (not visible) reflects the light, to a tertiary mirror, and finally to a small field steering mirror. Around the focal plane, each of the four instruments picks up the radiation from their corresponding field-of-view. The HERO pickoff mirror is slightly in front of the Origins field of view. It directs the light into an Offner relay ( 1 in Figs. 3 and 4), which has a movable mirror that can send the light to one of the four frequency bands. The cold receiver optics (2a) divides the sky signal into its two linear polarizations and reimages each onto a $3 \times 3$ focal plane mixer array.

There are eight LO chains 2 for each of the four frequency bands. To minimize coupling of the thermal radiation from the warm spacecraft bus to the cold Cryo-payload module (CPM), the eight LOs are combined into two beams in the warm LO optics (3): dichroics superimpose bands 1 on band 3 and band 2 on band 4 , and polarization grids superimpose the vertical polarization of band 1 plus 3 with the horizontal polarization of band 1 plus 3 and the same for bands 2 and 4 . (A design with four dichroics would be unable to provide continuous frequency coverage or have unacceptable losses.) The combined beams transfer the LO signals to the CPM, where the cold LO optics (2b) separates the LO beams into the four frequency bands and the two polarizations. A wire grid superimposes each LO signal onto a sky signal.

The LO and sky signals have orthogonal polarizations with respect to each other. Two mirrors and a lenslet array reimage the sky and the LO signals onto the focal plane mixer array. A set of $3 \times 3$ mixer feed horns captures the superimposed sky and the LO signal. A waveguide orthomode-transducer (OMT) at 45 degrees to both input signals divides the signals in sky plus LO and sky minus LO. These two signals are mixed and their outputs recombined. This method of superposition requires much less LO power than beam splitters, has no limitation on the IF bandwidth unlike Martin-Puplett interferometers, but there are some losses in the OMT. ${ }^{22}$ The HERO mixers consist of superconducting tunnel junction [superconductor-isolator-superconductor (SIS)] mixers or hot-electron bolometer (HEB) mixing junctions, depending on the target frequency band. A paper giving details of the HERO optics by Jellema is in preparation. 


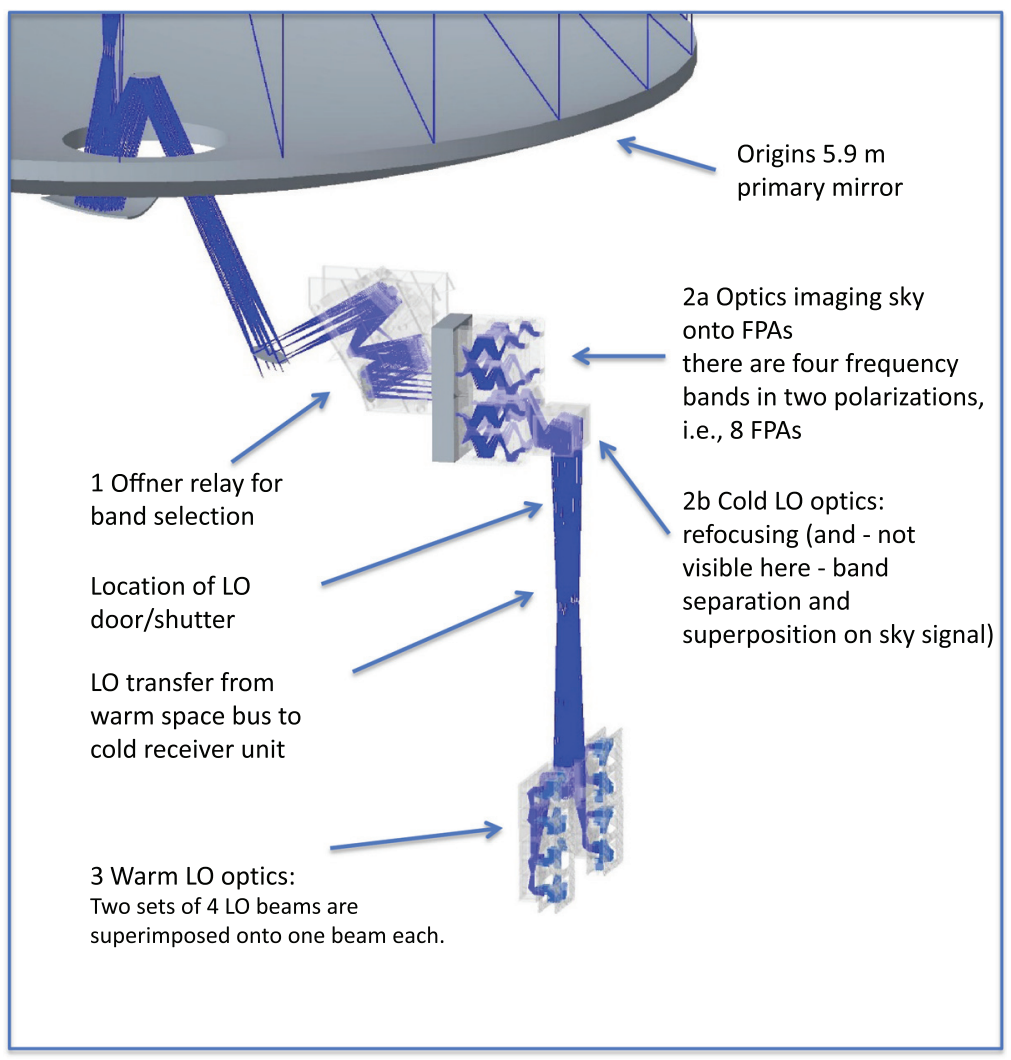

Fig. 3 Ray tracing of HERO optics. The signal from the sky is collected by the Origins antenna and redirected to the HERO instrument. The Offner relay mirror assures the band selection and sends the sky singal to one of the four frequency bands, where it is refocussed on two $3 \times 3 \mathrm{FPAs}$, one for each polarization. The LO signal comes from the spacecraft bus at the botton of the picture. A set of four LO beams are superimposed onto one optics paths and send to the cold instrument bay, where the LOs are refocused, separated and sent to the FPAs.

\subsubsection{Optical losses}

HERO optics has a throughput of $95 \%$, due to not perfect reflectivity and losses in the polarization grid, see Table 2. This calculation does not include the matching of the beams and truncation. We calculated the throughput of the HERO optics at its mid frequency of 1.6 tetrahertz $(\mathrm{THz})$, see Table 2. The HERO mirrors are gold plated and at $4.5 \mathrm{~K}$. Using the surface impedance $Z$ at $80 \mathrm{~K}$ for gold, the emissivity is $0.146 \%$ at $1.6 \mathrm{THz}$ (epsilon $=4 Z / Z_{0}$, with $Z_{0}=376.7 \Omega$ ). To account for slight surface roughness and misalignments, we doubled that number and used a mirror throughput of $99.7 \%$. For a wire grid using $10 \mu \mathrm{m}$ wire at $35-\mu \mathrm{m}$ spacing, Defrance ${ }^{23}$ calculates at $1.4 \mathrm{THz}$ a transmission of $99.8 \%$ at $0^{\circ}$ and $99.7 \%$ at $45^{\circ}$, for the reflections the numbers are slightly lower at $99.1 \%$ and $98.3 \%$, respectively. Jacob et al. ${ }^{24}$ obtained a good agreement between their calculations with measurements at $640 \mathrm{GHz}$, where transmission and reflections were above $99 \%$ for nearly all grids tested. For the HERO calculations, we used the most pessimistic number of $98.3 \%$. The lenslet array is an array of individual mirrors to refocus each beam onto the mixer array. We assumed a reflectivity of $99 \%$, worse than that of a single mirror. The Offner relay with the pick-off mirror consists of five mirrors (see Fig. 4, insert 1) and has thus a throughput of $98.5 \%$. The mixer optics (Fig 4, insert 2a) contains one wire grid, three mirrors, and the lenslet array and hence has a throughput of $96.4 \%$. Together, the HERO optics has $95 \%$ throughput.

The radiation is collected by corrugated feed horns. Munoz ${ }^{25}$ estimates the loss of the feed horn to be $-0.22 \mathrm{~dB}$ mainly due to losses in the walls of the waveguide, i.e., $95 \%$ throughput. Behind the feed horn is an OMT, which is expected to have about $-0.3 \mathrm{~dB}$ loss, i.e., $6.7 \%$. 


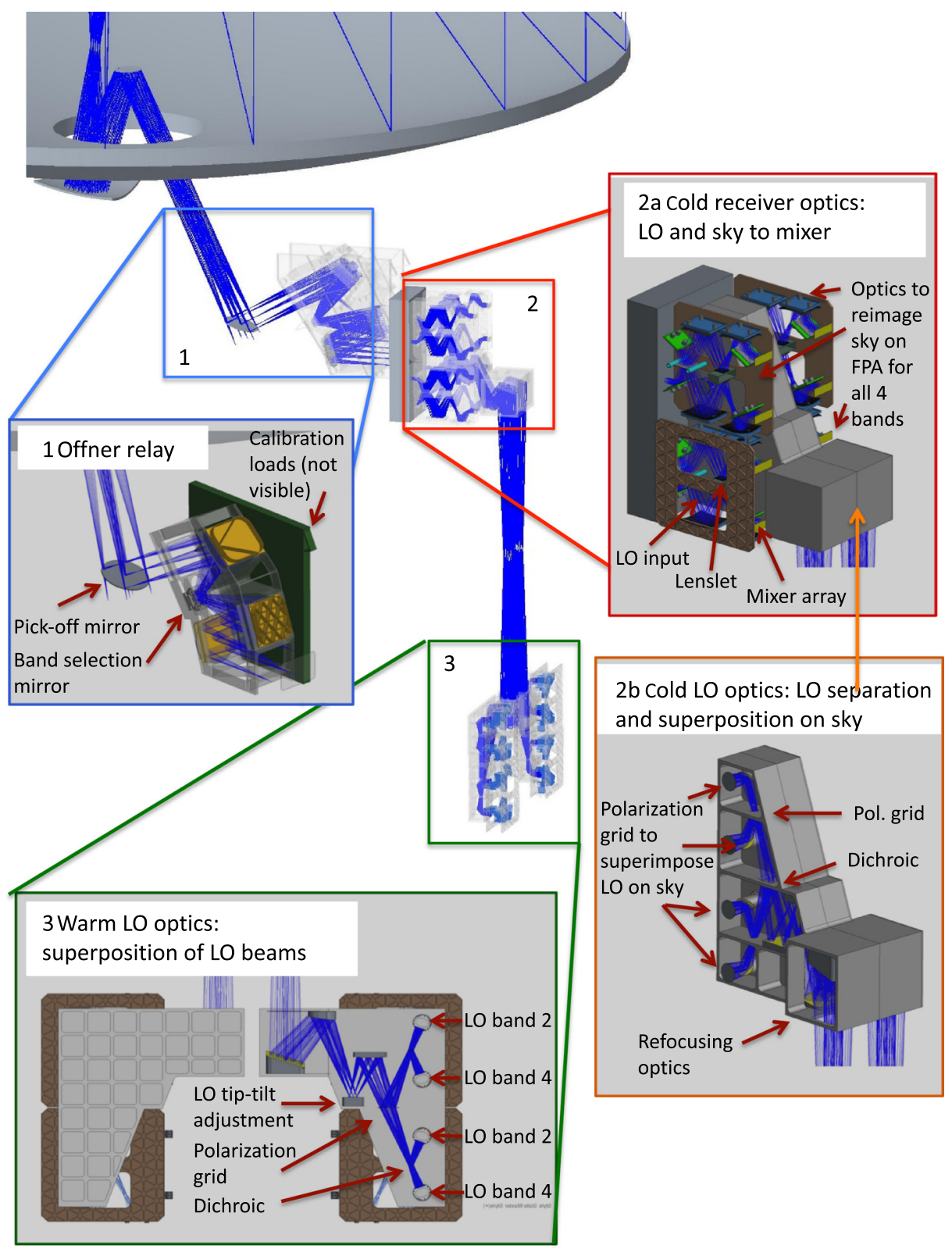

Fig. 4 HERO optics. Details of the Offner relay, the cold receiver optics with its cold LO optics and the warm LO optics.

Altogether, the optical losses and the losses in the feed horn and the OMT are expected to be $16 \%$.

Estimating the final aperture efficiency requires a diffraction calculation to determine the coupling efficiency of the corrugated feed horn to the primary mirror, via the whole optics system.

\subsubsection{Challenges}

The greatest challenges of optics design are the wide bandwidth coverage of nearly three octaves, which requires a nearly frequency-independent design. A lenslet array in the cold receiver optics is used to map the sky, and the LO to fit the spacing of the mixers in the FPA. This component needs careful alignment, and optimization in the design phase.

Another challenge is the LO transmission from the warm spacecraft bus to the mixers, that are over $2 \mathrm{~m}$ away. 
Table 2 HERO optical throughput.

\begin{tabular}{llc}
\hline \hline Optical element & \multicolumn{1}{c}{ Specification } & $\begin{array}{c}\text { Optical } \\
\text { throughput (\%) }\end{array}$ \\
\hline Mirror & Assumption: gold plated, $4.5 \mathrm{~K}$ (theoretical calculation $\times 2)$ & 99.7 \\
Wire grid & Assumption: $10 \mu \mathrm{m}$ wire, 35- $\mu \mathrm{m}$ spacing, 45 deg angle & 98.3 \\
Lenslet array & & 99 \\
Horn & Corrugated & 95 \\
OMT & Finline OMT & 93.3 \\
Offner relay & 5 mirrors (see Fig. 4) & 98.5 \\
Mixer optics & Three mirrors, one wire grid, one lenslet array & 96.4 \\
Sum HERO optics & Offner relay, mixer optics & 95 \\
HERO optics plus & Offner relay, mixer optics, horn, and OMT & 84 \\
horn and OMT & & \\
\hline \hline
\end{tabular}

As mechanical stability between the warm spacecraft bus and the cold instrument cannot be guaranteed, the warm LO optics has a tip-tilt mirror (see Fig. 4, insert 3) that can be adjusted to maximize the LO power on the mixer.

In addition, the IR heat transfer needs to be kept minimal. In addition to superimposing the eight LOs onto two combined LO beams, these combined LO beams are kept as narrow as possible and the beam passes through 5 thermal blocking filters. These bandpass filters need to provide nearly lossless transmission of the LO signal, but block any out of band IR radiation efficiently. A single heat sunk filter is able to block out of band radiation to $-23 \mathrm{~dB}$ and thus the 5 filters reduce the heat load to $2.4 \mathrm{~mW}$. An alternative solution would be low loss fiber-like waveguides for 500 to $2700 \mathrm{GHz}$, with a loss of $<2 \mathrm{~dB}$ per m. Currently, these fibers do not exist yet; for an overview see, e.g., Humbert. ${ }^{26}$

\subsection{Local Oscillators}

Eight LO chains consisting of a combination of amplifier and multiplier stages multiply the frequency of a low noise W-band synthesizer signal to the required target LO frequency. ${ }^{27,28}$ The LO signal is divided by waveguide power divider into nine outputs to match the mixer arrays, similar to the 16 beam example shown in Fig. 5.

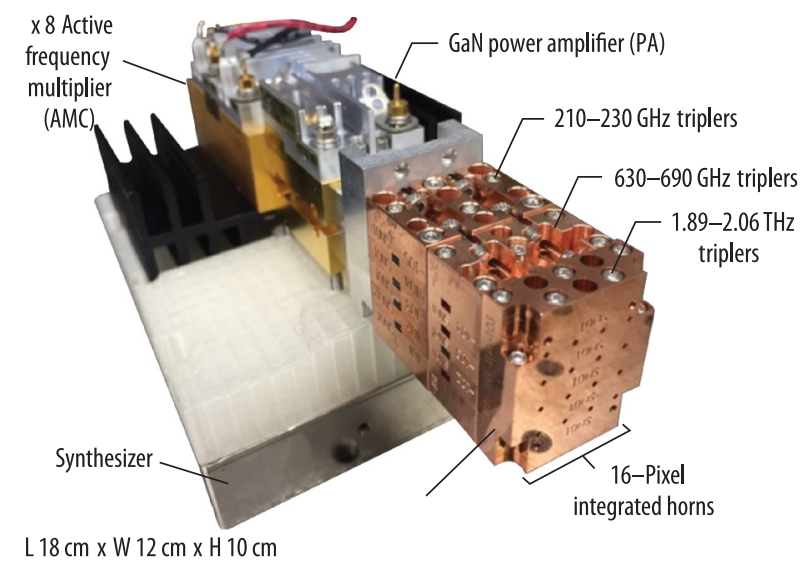

Fig. 5 Compact multi-pixel electronically tunable LO systems have been demonstrated to 1.9 THz. The system shown here multiplies a $\sim 9 \mathrm{GHz}$ signal to $\sim 70 \mathrm{GHz}$, amplifies it, then splits it into 16 signals and then further multiplies each to $\sim 1.9 \mathrm{THz}$. The output is a 16 beam LO signal that can directly pump 16 mixers in a FPA. HERO has a similar chain with 9 outputs. 
The HERO LOs have bandwidth of nearly $50 \%$ each to reduce the number of LOs and hence weight and volume in the spacecraft. Broadband amplifiers and multipliers are difficult to design, but multipliers for submillimeter LOs with $40 \%$ bandwidth have been already demonstrated, e.g., Virginia Diodes (VDI). ${ }^{29}$ HERO requires extremely efficient multipliers as we have only allocated $2 \mathrm{~W}$ of DC power to each amplifier chain. The design of amplifier multiplier chains up to $2.7 \mathrm{THz}$ with sufficient power to pump an array of mixers is difficult, but the prototype chains already exist for single-pixel applications, e.g., Mehdi et al. ${ }^{27}$ for an overview, $\mathrm{VDI}^{28}$ with $5 \mu \mathrm{W}$ at $2.7 \mathrm{THz}$. An alternative approach for the LO source is the Quantum Cascade Laser (QCL). ${ }^{30-33}$ These components have sufficient output power, but require cooling and generally have very limited tunability. Hübers ${ }^{34}$, and Hübers et al. ${ }^{35}$ have packaged a QCL with $0.6 \mathrm{~mW}$ output power in a compact cooler requiring $85 \mathrm{~W}$ input power. For continuous tuning, a multimode QCL with a frequency selecting Fabry-Pérot interferometer has been developed. ${ }^{36}$

\subsection{Mixer}

HERO uses the most sensitive mixers available. SIS mixers ${ }^{37-39}$ are utilized in the FPAs at the two lower frequency bands 486 to $756 \mathrm{GHz}$, and 756 to $1188 \mathrm{GHz}$. HERO's SIS mixers will have noise temperatures of $2 h f / k$ ( $h=$ Planck constant, $f=$ frequency, and $\mathrm{k}=$ Boltzmann constant), which has been already achieved for HIFI/Herschel ${ }^{15}$ for band 1 . HEB ${ }^{40-43}$ mixers make up the FPAs for the bands 1188 to $1782 \mathrm{GHz}$ and 1782 to $2700 \mathrm{GHz}$. The HEBs will have noise temperatures below $4 \mathrm{hf} / \mathrm{k}$. Currently, the upGREAT ${ }^{14}$ Instrument on SOFIA demonstrated the best noise temperatures of 700K double sideband (DSB) at $1.9 \mathrm{THz}$, and HEBs are continuously improving, with a $780 \mathrm{~K}$ receiver noise temperature (all components included) measured at $2.7 \mathrm{THz}{ }^{44}$ An overview of mixer noise temperatures is given in Fig. 6.

HERO has eight FPA (four frequency bands with two polarizations each). Each FPA consists of $3 \times 3$ mixers in a square configuration. The SIS mixers that require a magnetic field have a footprint of $10 \times 10 \mathrm{~mm}^{2}$, and the HEBs $5 \times 5 \mathrm{~mm}^{2}$. Projected onto the sky, the arrays have $2 \times$ full width half maximum beam spacing, which is the closest spacing that can be achieved without considerable cross-talk between pixels and low antenna efficiency. A similar FPA with $4 \times 4$ pixels is shown in Fig. 7 .

Eight mixers of each array are DSB balanced mixers. The balanced mixer design is adopted to reduce the LO power required and to enhance the stability by suppressing LO amplitude modulation noise. ${ }^{42}$ One mixer in each FPA is a sideband separating (2SB) mixer to allow sideband calibration of the DSB mixers. Each junction can be individually DC biased for optimal operation.

The baseline IF range is 0.5 to $6.5 \mathrm{GHz}$ (goal of 0.5 to $8.5 \mathrm{GHz}$ ) so that the IF covers at least $670 \mathrm{~km} / \mathrm{s}$ at the highest frequency, sufficient for observations of the inner Milky Way and of nearby galaxies. This IF bandwidth is typically not a problem for SIS mixers but is challenging for HEBs. A priori, NbN HEBs will be used, because NbTiN HEB mixers have narrower IF bandwidth (3 GHz), NbN HEBs need more LO power but have wider IF bandwidth.

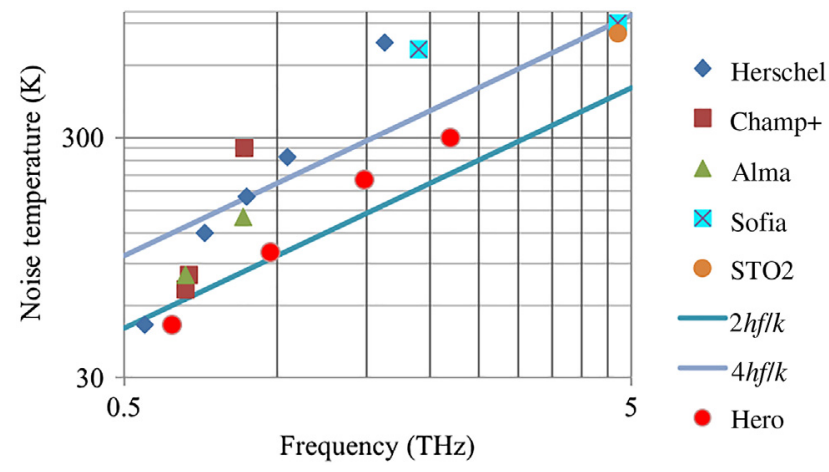

Fig. 6 DSB receiver noise temperature of different telescopes and the HERO goal. Below $1 \mathrm{THz}$ the SIS mixers have nearly reached the requirement of $2 \mathrm{hf} / \mathrm{k}$. HERO goal of $2 \mathrm{hf} / \mathrm{k}$ up to $1188 \mathrm{GHz}$ and $4 \mathrm{hf} / \mathrm{k}$ at higher frequencies. HEB mixers development is still required. 


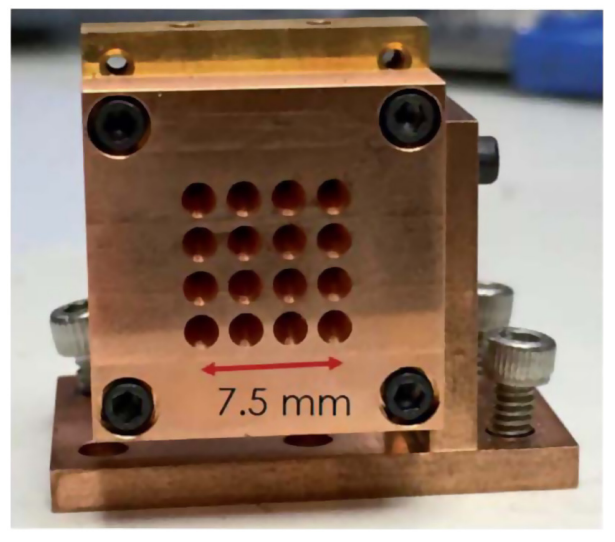

Fig. 7 A 16-element 1.9 THz mixer array with smooth-walled spline feed horns in a square grid configuration. The block, including circular-to-rectangular waveguide transitions and pockets to hold the hot-electron-bolometer mixers, was directly machined from copper. ${ }^{45}$

Recently, NbN HEB mixers on a GaN buffer-layer operating at $\sim 1.3 \mathrm{THz}$ with $7.5 \mathrm{GHz}$ IF bandwidth have been demonstrated, ${ }^{46}$ and efforts to increase the IF bandwidth are ongoing. An alternative are $\mathrm{MgB}_{2}$ mixers ${ }^{47}$ that have IF bandwidth of more than $8 \mathrm{GHz}$, however, they do not have the same sensitivity, yet $(1400 \mathrm{~K}$ at $1600 \mathrm{THz})$, but progress is being made. The common Origins closed-cycle cryocoolers cool the mixers to about $4.5 \mathrm{~K}$.

\subsection{Intermediate Frequency and Backend}

The IF signal at the output of the mixer is immediately amplified by $\sim 20 \mathrm{~dB}$ by an LNA at $4.5 \mathrm{~K}$ physical temperature, then again by $\sim 20 \mathrm{~dB}$ at a physical temperature of $35 \mathrm{~K}$, and finally in the warm spacecraft bus. The first stage low-noise $(<6 \mathrm{~K})$, low-power SiGe cryogenic amplifiers ${ }^{48-50}$ [Fig. 8(a)] are directly behind the mixer arrays and connected to the mixers by a short transmission line on a flexible Mylar bus. ${ }^{52,53}$ Low power dissipation of the LNAs is essential to prevent the mixers from heating up and to minimize the cooling requirements. Bardin et al. ${ }^{48}$ have recently demonstrated a state of the art SiGe amplifier integrated with a SIS mixer having an instantaneous bandwidth of $4 \mathrm{GHz}$ and with power dissipation as low as $0.3 \mathrm{~mW}$. An alternative are the mature InP High-Electron-Mobility Transistor (HEMT) amplifiers, ${ }^{54}$ which have already been used on Herschel. Normally, InP amplifiers would require around 5-mW power per pixel, but tests operating at only $0.5 \mathrm{~mW}$ have shown only slightly higher noise temperatures. ${ }^{55}$ If InP amplifiers operating with less than the nominal power are stable, they could serve as a backup alternative to SiGe.

Passive power combiners multiplex the IFs of different bands, such that the $2 \times 9$ pixels of any two frequency bands can be operated simultaneously. The multiplexing minimizes IF cables and back-ends, thus economizing on weight and thermal conduction. Coaxial cables carry 40 IF channels to the $35 \mathrm{~K} \mathrm{LNAs}$ for further amplification, and then to the spacecraft bus. Miniaturized

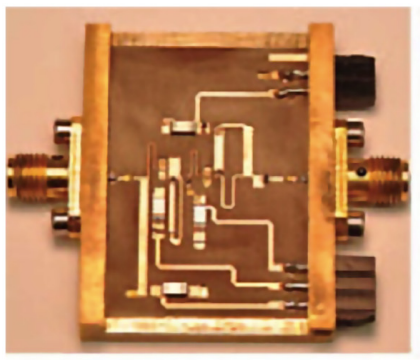

(a)

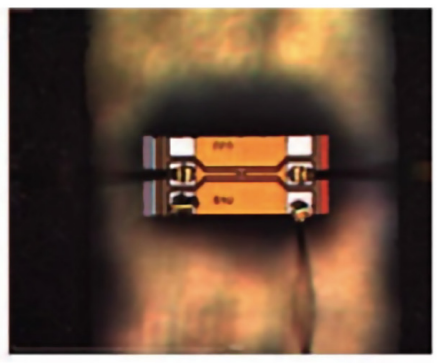

(b)

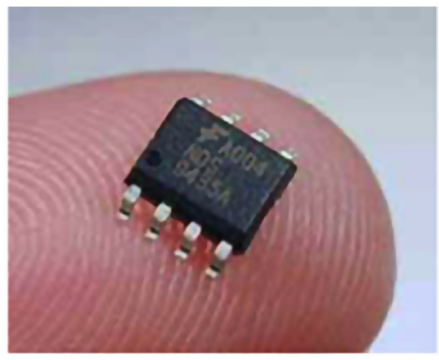

(c)

Fig. 8 (a) Low power SiGe cryogenic amplifiers. ${ }^{50}$ (b) and (c) Extremely compact CMOS prototype amplifiers. $^{51}$ 
integrated IF circuits ${ }^{51}$ [Figs. 8(b) and 8(c)] further amplify, filter, and condition the signal for the backends.

HERO requires 40 low-power $(\sim 1 \mathrm{~W})$ spectrometers, each covering a bandwidth of 6 to $8 \mathrm{GHz}$. Complementary Metal Oxide Semiconductor (CMOS) Application-Specific Integrated Circuit (ASIC) backends as developed by Tang et al. ${ }^{56,57}$ and others have very low power consumption, thousands of spectral channels and are rapidly advancing in bandwidth. ${ }^{58,59}$ The spectrometers contain analog-to-digital converters, FFT processors, and data accumulators. They digitize to 3- or 4-bit resolution, sufficient for our application. Alternatively, HERO could use backends based on field-programmable gate array (FPGA) technology ${ }^{60,61}$ They show excellent performance, but their power consumption has to be lowered from currently around $70 \mathrm{~W}$ to about $2 \mathrm{~W}$ per spectrometer to be usable in HERO.

To increase the frequency resolution and minimize spectral leakage, HERO will use window functions in the spectrometer such as Blackman-Harris windows that reduce the side lobes to below $-61 \mathrm{~dB}$. The HERO spectrometer will take full advantage of prior experiments and knowledge, described e.g., by Klein et al. ${ }^{62}$

\subsection{Control Electronics}

There are three different control units, one for each subsystem to make testing before integration easier. The LO control unit monitors and controls the synthesizer and the LO Unit with the amplifier multiplier chains. The focal plane control unit monitors and controls all the components mounted at $4.5 \mathrm{~K}$, in particular, the mixer bias, the amplifier bias, and the Offner relay mirror that is used to select between the different frequency bands. It also monitors and regulates the temperature of the calibration loads. The instrument control unit (ICU) monitors and controls the IF and the spectrometers. This unit also reads the data from the spectrometers and compresses it before passing it to the Origins bus computer for downlinking. The ICU is the interface between the Origins bus computer and the other control units of the HERO instrument.

The control units use state of the art onboard processors such as the GR740 rad-hard quadcore LEON4FT. They use spacewire as an interface between the units and MIL1553STDB as an interface to the spacecraft. All control units are mounted on the spacecraft bus in pairs, one operating unit and one redundant spare that is normally not operating.

\section{Thermal and Mechanical Design and Mechanisms}

\subsection{Thermal Design}

The thermal design contains on one hand requirements for the instrument to operate correctly and on the other hand the requirement that the instrument's heat load on the satellite should be minimized. The HERO front-end is located in the CPM at $4.5 \mathrm{~K}$, the LOs, back-end, and the control units in the warm $(\sim 300 \mathrm{~K})$ spacecraft bus. The CPM is cooled by four cryocoolers, which can each lift $50 \mathrm{~mW}$ of heat, i.e., there is only $200 \mathrm{~mW}$ cooling power available in the CPM for all instruments, mechanical structures, and thermal radiation.

The only components that require cryogenic temperatures for operation are the superconducting mixers. They only work below $\sim 6 \mathrm{~K}$ and have slightly better performance at lower temperatures. The mixer mounts have brass straps to two of the cold fingers of the ISM and hence are cooled to $4.5 \mathrm{~K}$. A Mylar cable bus separates the mixers from the LNAs, which have significant heat dissipation of around $0.5 \mathrm{~mW} /$ amplifier. The second components that need to be cooled are the LNAs, which add less noise to the signal when cold. There is no strict temperature limit, so we have compromised between instrument performance and cooling demands on the satellite. The outcome is that the first stage LNAs with $20 \mathrm{~dB}$ gain are on the CPM at slightly above $4.5 \mathrm{~K}$, the second stage with another $20 \mathrm{~dB}$ gain at $35 \mathrm{~K}$, and the third stage with $60 \mathrm{~dB}$ gain in the warm spacecraft bus. The emissivity of reflective optics elements at submillimeter wavelengths is small (5\%, see Table 2), and since the noise temperatures are 50 to $300 \mathrm{~K}$, the temperature of the optics has only a small impact on the system noise temperatures.

The second design criterion is to keep the cooling demands of the satellite low. On the $4.5 \mathrm{~K}$ stage, the LNAs are the most important heat source. We have selected SiGe amplifiers, ${ }^{48}$ which 
can operate at around $0.3 \mathrm{~mW}$ for $20 \mathrm{~dB}$ gain, as opposed to InP $\mathrm{LNAs}^{53}$ as used in HIFI/ Herschel, which had $5 \mathrm{~mW}$ power dissipation. All amplifiers not in use are turned off.

The passive heat load on the $4.5 \mathrm{~K}$ stage comes from three major sources: the warm calibration load, the two holes through which the LO beams pass, and the cable harness. We carefully designed a thermally suspended calibration load with a shutter that can be closed when HERO is not in operation. We minimized the IR radiation through the LO beam by adding five heat sunk bandpass filters that block all IR radiation except that in the observing range of HERO. The filters reduce the heat load to $2.4 \mathrm{~mW}$ per beam, i.e., $4.8 \mathrm{~mW}$ in total. In addition, the LO beams have a shutter that is closed when HERO is not in operation to minimize stray light to the other instruments. In the cable harness, the coaxial IF cables conduct the most heat. We have designed a passive multiplexing system on the $4.5 \mathrm{~K}$ stage so that only $40 \mathrm{IF}$ signals for the $2 \times 2 \times(9+1)$ beams are carried back to the spacecraft bus $(+1$ because one mixer in each FPA is sideband separating and has a second IF signal). The cable harness is heat sunk on the $35 \mathrm{~K}$ stage.

The thermal design on the spacecraft bus is less critical, because the operating temperature range is larger between $\sim 200 \mathrm{~K}$ and $320 \mathrm{~K}$, and heat can be removed by radiators. Nevertheless, we strive to use components with low power consumption, in particular spectrometers requiring only $2 \mathrm{~W}$ of power each, and efficient LO frequency multiplier chains. Alltogether HERO requires $205 \mathrm{~W}$ of power.

\subsection{Mechanical Design}

Figure 9 shows a computer-aided design (CAD) drawing of HERO with the mechanical components in gray, brown, and green, and the ray trace in blue. The Offner relay and the cold receiver optics including the cold LO optics fit in an envelope of $1.05 \times 1.47 \times 0.4 \mathrm{~m}^{3}$. The warm LO optics requires $1.37 \times 0.8 \times 0.21 \mathrm{~m}^{3}$. Given the large space of the CPM under the 5.9 m primary mirror HERO fits comfortably between the other instruments as can be seen in Fig. 10.

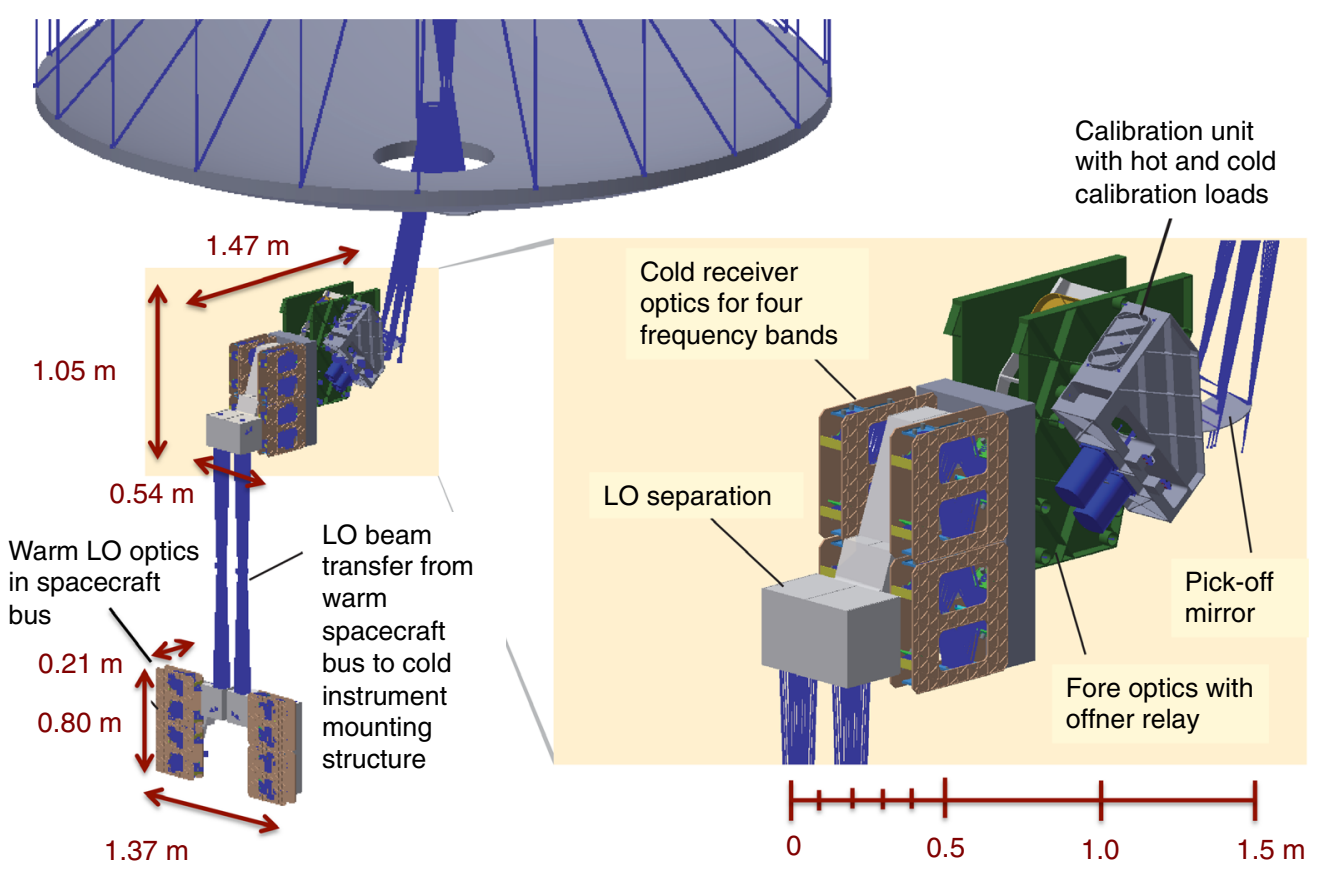

Fig. 9 CAD drawing of HERO with ray trace in blue. The Offner relay, the cold receiver optics, and the LO separation optics are at $4.5 \mathrm{~K}$ and are in the CPM below the primary mirror in the upper part of the figure on the left and in the enlarged drawing on the right. To minimize the cryogenic heat load, the LO signals are generated in the spacecraft bus. The warm LO optics (lower part of figure) superimpose the $8 \mathrm{LO}$ signals onto two beams and transfer them from the warm spacecraft bus the cold CPM. 


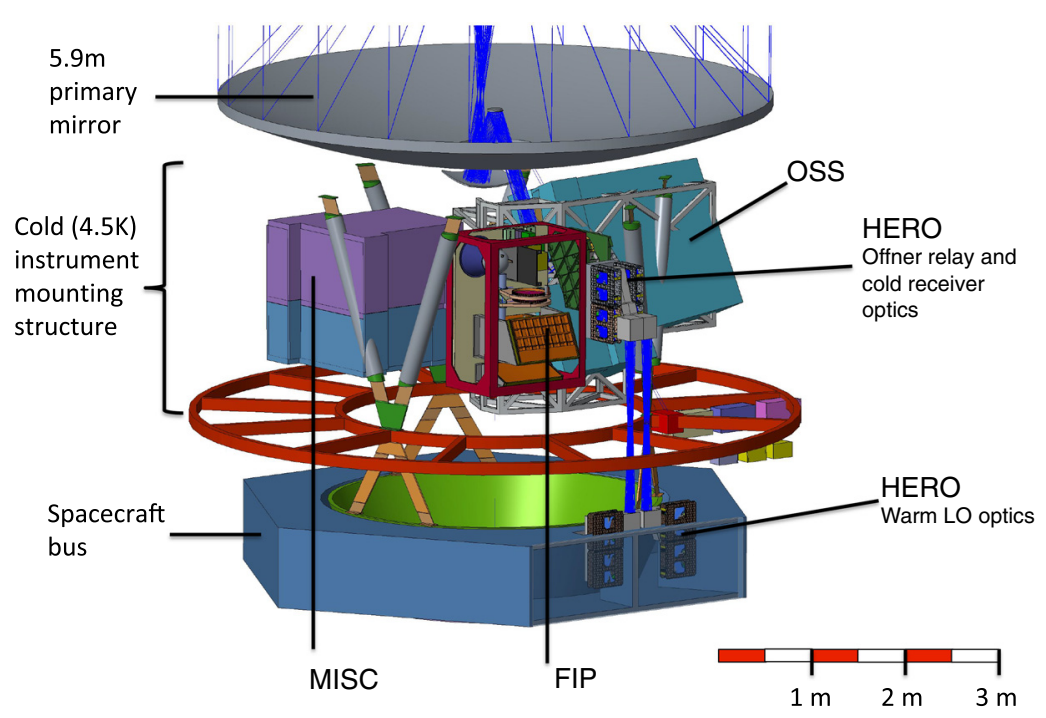

Fig. 10 HERO fits comfortably into the spacecraft between the other three instruments, MISC, FIP, and OSS.

Despite using lightweight mechanical structures, HERO still weighs $\sim 180 \mathrm{~kg}$. The mirrors for the LO relay and their support structure are the biggest contributors weighing $40 \mathrm{~kg}$.

\subsection{Mechanisms}

HERO has only four mechanisms (Table 3). The Offner Relay ${ }^{63-66}$ directs the sky beam to the respective frequency array with the help of a band selection mirror (see Fig. 4, insert 1) that has two axis of rotation. The tip-tilt mirror (Fig. 4, insert 3) on the warm side of the LO relay compensates for any mechanical displacement between the warm spacecraft bus and the focal plane. Finally, the calibration load shutter and the LO door [location of LO door see Fig. 3, for the design see light tight shutter design for SPace Infrared telescope for Cosmology and Astrophysics (SPICA) ${ }^{67}$ ] can be closed when HERO is not in use to minimize heat transfer and to ensure a dark environment for the other instruments. All mechanisms are based on prior designs and are hence relatively low-risk.

\subsection{HERO Tests, Integration, Alignment, and Calibration}

The Herschel/HIFI testing, integration, alignment, and calibration procedures (see $\mathrm{PhD}$ thesis by Jellema ${ }^{68}$ ) will be used for HERO. The HERO instrument team tests, calibrates, and

Table 3 HERO uses two proven mechanisms and two shutters.

\begin{tabular}{|c|c|c|c|}
\hline Mechanism & Function & Accuracy & Heritage \\
\hline Offner relay & Select frequency band, calibration load & $\begin{array}{l}1 \text { '30" accuracy, } \\
15 \text { deg range }\end{array}$ & Herschel/SPIRE63 \\
\hline Tip-tilt for LO & $\begin{array}{l}\text { Mirror allowing to adjust Lo beam for mechanical } \\
\text { drift between the space craft bus and the cold } \\
\text { instrument unit }\end{array}$ & $1 "$ accuracy & $\begin{array}{l}\text { Herschel/HIFI } \\
\text { (focal plane } \\
\text { chopper }^{64} \text { ) }\end{array}$ \\
\hline $\begin{array}{l}\text { Calibration load } \\
\text { shutter }\end{array}$ & Cover calibration load to minimize heat load & $\begin{array}{l}1 \mathrm{~mm} \text { at } 50-\mathrm{mm} \\
\text { base rotational } \\
\text { cover }\end{array}$ & Herschel/HIFI \\
\hline LO door/shutter & $\begin{array}{l}\text { Close optical connection between LO units in } \\
\text { warm spacecraft bus and cold telescope }\end{array}$ & $\begin{array}{l}1 \mathrm{~mm} \text { at } 50-\mathrm{mm} \\
\text { base rotational } \\
\text { cover }\end{array}$ & Herschel/HIFI \\
\hline
\end{tabular}


Table 4 The HERO instrument summary.

\begin{tabular}{|c|c|}
\hline HERO & Current best estimate \\
\hline Mass & $183 \mathrm{~kg}$ \\
\hline Volume & $1.28 \mathrm{~m}^{3}$ \\
\hline Power & $205 \mathrm{~W}$ \\
\hline Heat dissipation ${ }^{\mathrm{a}}$ at $4.5 \mathrm{~K}$ & $20 \mathrm{~mW}$ \\
\hline Heat dissipation ${ }^{\mathrm{a}}$ at $35 \mathrm{~K}$ & $40 \mathrm{~mW}$ \\
\hline Date rate & $5.1 \mathrm{kbit} / \mathrm{s}$ \\
\hline TRL in 2020 & $\geq 4$ \\
\hline TRL in 2027 & $\geq 6$ \\
\hline
\end{tabular}

internally aligns the instrument in existing cryostats prior to delivery. Origins is designed such that each instrument can be removed separately for maintenance and upgrades. Consequently, the National Aeronautics and Space Administration (NASA) team can integrate the instruments in any order.

The usual procedures are used to calibrate HERO in flight: HERO has two internal calibration loads to calibrate the receiver response, one of the mixers in each array is sideband separating to allow a calibration of the sideband ratio of the DSB mixers, and calibration on astronomical continuum sources (such as planets) are used to obtain a bandpass and allow the determination of the optical coupling/ aperture efficiency. Observations of point sources are used to determine the main beam efficiency.

HERO has two calibration loads, a hot load at a few tens of $\mathrm{K}$ and a cold load at $4.5 \mathrm{~K}$. The loads are made of stycast or a similar material that looks like a black body at THz frequencies. The loads are cone shaped to have many internal reflections and to avoid any backscattering into the receiver. To minimize any heating of the CPM, the loads have infrared filters. The filters are mounted at an angle to avoid reflection and standing waves. The loads also have a shutter that will be closed when HERO is not in operation.

\subsection{Resource Requirements}

Table 4 summarizes the resources required by HERO.

\section{Performance}

The HERO has near quantum-limited performance and has unrivaled sensitivity over a very large frequency range (see Fig. 11). It consists of small mixer arrays, observes in both polarizations simultaneously and allows dual-frequency observations. It uses very little power per pixel and is the first heterodyne array receiver designed for space. The size of the HERO FPAs are well matched to prestellar cores thus requiring generally only a single pointing. For point source observations, HERO is 5 to 20 times more sensitive than Herschel/HIFI, i.e., can observe a factor of 25 to 400 times faster. This is partly due to the large collecting area of the Origins $5.9-\mathrm{m}$ primary mirror (versus $3.5 \mathrm{~m}$ for Herschel) and partly due to improved sensitivity especially of the HEB mixers (e.g., at $1.9 \mathrm{THz}$ HERO has $300 \mathrm{~K}$ receiver temperature versus $1100 \mathrm{~K}$ for HIFI). We used an aperture efficiency of 0.8 for Origins, versus 0.65 for Herschel. When mapping extended sources convolved to the same beam size, HERO is 10 to 30 times more sensitive, i.e., 100 to 900 times faster than HIFI/Herschel. HERO can map so much faster because it has 9-pixel arrays, can do dual-frequency observations and has more sensitive mixers, especially at the high frequencies. Being on a satellite, the full FIR spectrum will be available to HERO, 


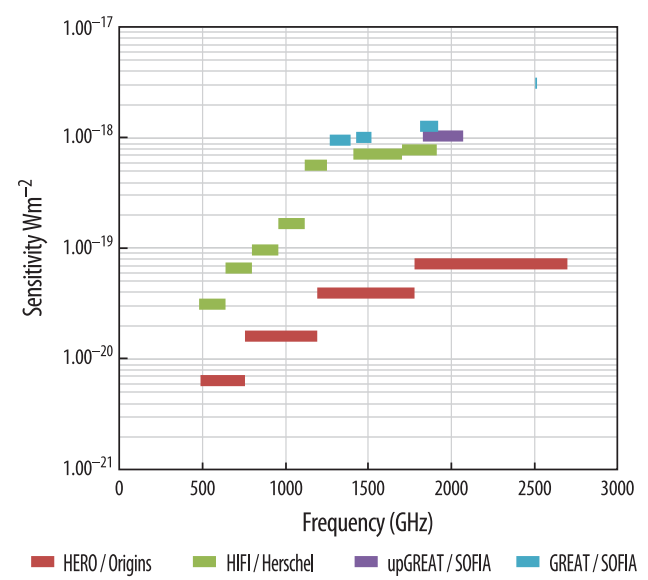

Fig. 11 HERO is more sensitive by an order of magnitude than any prior heterodyne instrument and has the widest frequency coverage of any instrument. It is worth noting that the sensitivity calculations for SOFIA assume a perfectly transparent atmosphere, which is not true close to the water lines.

whereas ground based telescopes and even SOFIA operate behind a more or less opaque curtain produced by atmospheric absorption.

\section{Technology Roadmap}

HERO builds on the current state-of-the-art receivers but surpasses them in different aspects of performance. For the current design, the latest and most promising Research and Development $(\mathrm{R} \& \mathrm{D})$ results have been taken into account.

Table 5 gives an overview of prior missions, the HERO design and the technical readiness level (TRL) of the HERO components. Concerning the LO, multiplier amplifier chains have been used for HIFI/Herschel, however, the HERO LOs need to have wider relative bandwidth, higher efficiency to pump nine mixers and need to reach $2.7 \mathrm{THz}$ versus $1.9 \mathrm{THz}$ for HIFI; we hence consider that this proven technology has TRL 5. SIS, and HEB mixers have flown on HIFI/ Herschel. HERO will have arrays of mixers, however these are essentially closely packed individual mixers, so no difficulty is expected. HERO will have more sensitive mixers, in particular more sensitive HEB mixers than HIFI/Herschel. More sensitive mixers are already used in upGREAT on SOFIA, though some improvement is still needed for HERO. Up-GREAT has already demonstrated mixers in waveguide up to $4.7 \mathrm{THz}$, so this technology can be used for HERO up to 2.7 THz. Herschel has used InP LNAs, but Stratospheric TeraHertz Observatory (STO) ${ }^{69}$ and GREAT have used SiGe; both worked well. For HERO we need the SiGe LNAs but with lower power consumption and space qualified (TRL 4). All missions have so far flown digital FFTs. CMOS-based system on chip (SOC) have been characterized and tested in the laboratory and have the required bandwidth and power, but they still need to be space qualified (TRL 5).

Table 6 lists the risks and mitigations that could be taken if these goals are not all met. In summary, HERO is a very low risk mission. In the design, we pushed current technologies further and used come innovative components. However, if any of these components should not be ready, there is always the HIFI/Herschel technology to fall back on. This would allow us to either built HERO with less pixels, or to build the HERO described here but with higher cooling power, electrical power, and mass budgets.

It is essential to carry out R\&D work on components to ensure the HERO mission, we envisage. Figure 12 summarizes the technology roadmap for HERO.

\section{EHT Extension}

The concept of using the Origins Space Telescope (Origins) to study black hole physics on event horizon scales has recently been raised as a potential extension of the HERO science case. ${ }^{70}$ 
Wiedner et al.: Heterodyne Receiver for Origins

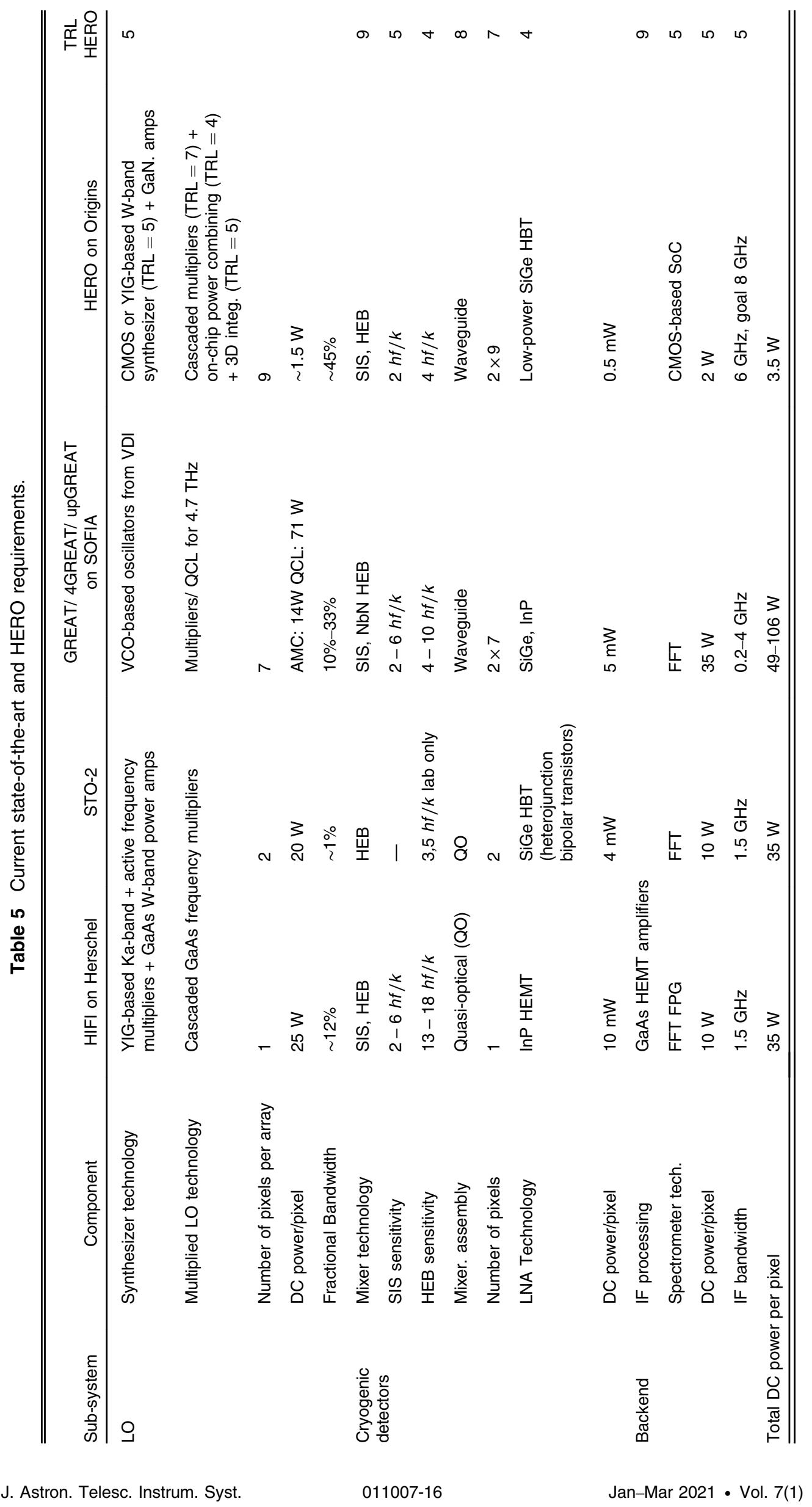


Wiedner et al.: Heterodyne Receiver for Origins

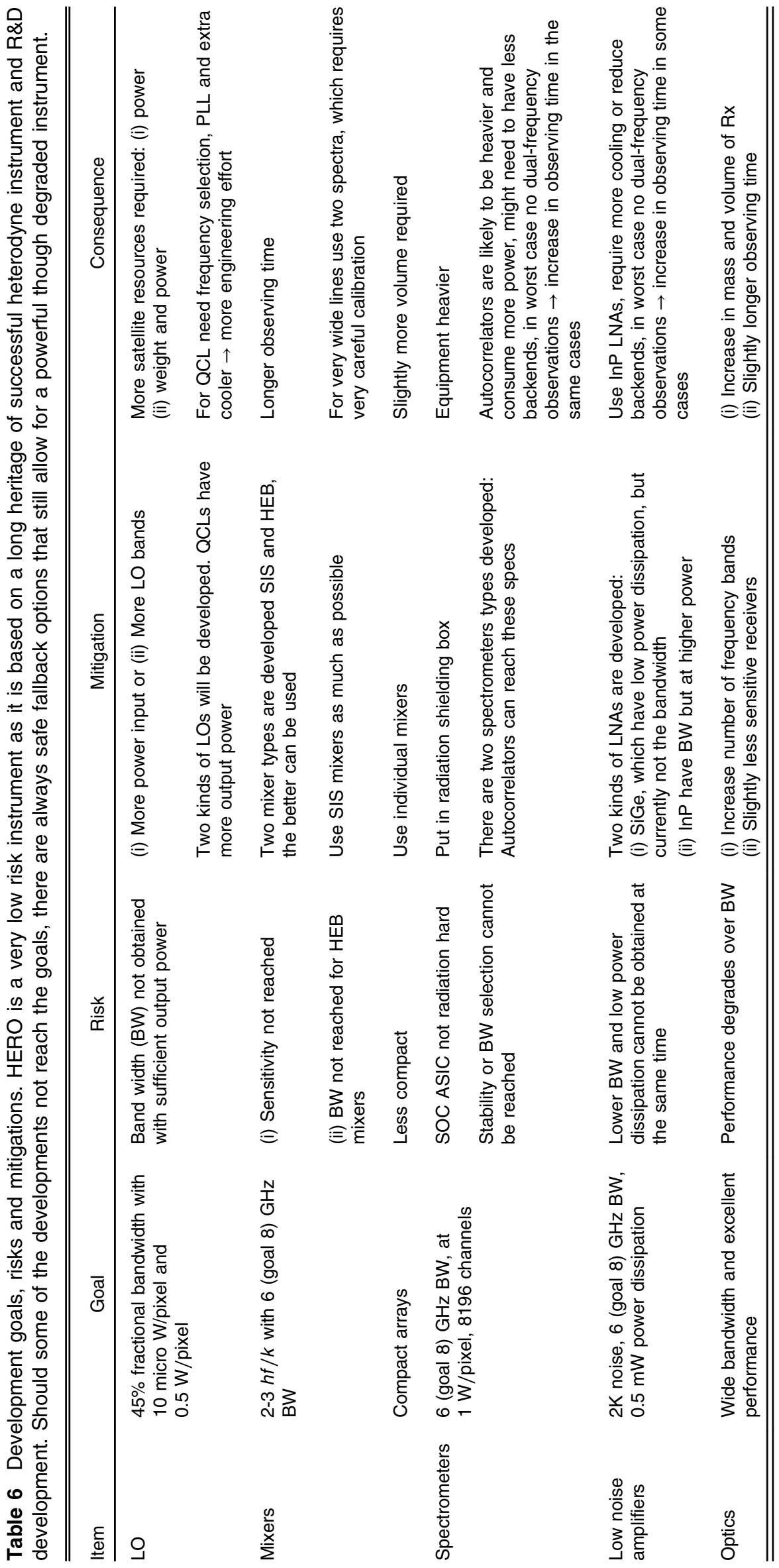




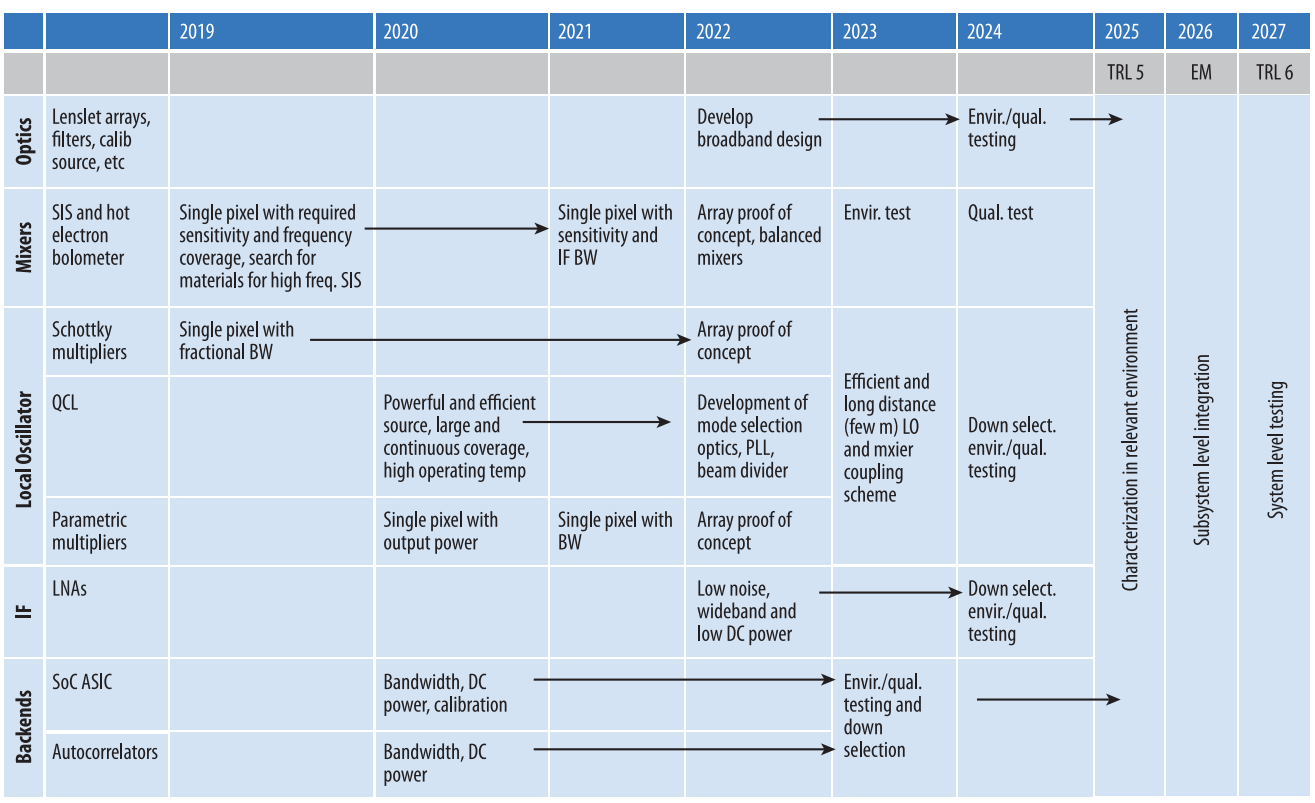

Fig. 12 The development schedule ensures a low-risk technology development roadmap for HERO.

Preliminary science concept exploration shows much promise and merits further study. The unprecedented angular resolution resulting from the combination of Origins with existing ground-based submillimeter/millimeter telescope arrays would increase the number of spatially resolvable black holes by a factor of $10^{6}$, permit the study of these black holes across all cosmic history, and enable new tests of general relativity by unveiling the photon ring substructure in the nearest black holes. Expanding the HERO instrument to be an interferometric station will require adding broadband single pixel receivers at $86,230,345$, and $690 \mathrm{GHz}$, a very accurate time reference, fast on-board data processing, and a fast downlink. A top-level examination of these requirements has not revealed any insurmountable obstacles to achieving this goal.

\section{Conclusion}

The HERO is the first heterodyne FPA designed for space. It builds on the solid foundations of prior heterodyne receivers and in particular on the successful Herschel/HIFI instrument. HERO incorporates the latest mixer developments to reach unprecedented sensitivity. Using innovative components and a compact design, HERO demands only relatively modest resources of the satellite. HERO also surpasses any prior instrument in terms of wavelength coverage and observing efficiency; it allows dual polarization and simultaneous dual-frequency observations with four 9-pixel arrays. HERO will help to map the trail of water from the ISM to habitable worlds. With its unique capacities HERO opens a new window onto the physics and chemistry of the ISM and invites observers to explore the still largely unknown FIR Universe.

\section{Acknowledgments}

The authors are grateful to the many institutions that sponsored and contributed to the successful study of the HEterodyne Receiver for Origins, as well as the individuals that dedicated their time and effort. In particular, the Centre National d'Etudes Spatiales (CNES), SRON Netherlands Institute for Space Research (SRON), and the Swedish National Space Agency have supported this study. Part of this research was carried out at the Jet Propulsion Laboratory, California Institute of Technology, under a contract with the National Aeronautics and Space Administration. We would like to thank SRON, NOVA at ASTRON, and the Observatoire de Paris for providing engineering support for the optical and mechanical design. The study would never 
have taken place if NASA had not invited international members to participate in the Origins Science and Technology Definitions Team (STDT). The help and support of the NASA engineering team was very much appreciated. We would like to thank everybody on the Origins and HERO team for a fantastic study, interesting science, great engineering ideas, hard work, and the friendly and cooperative atmosphere.

\section{References}

1. M. Meixner and A. Cooray, "Origins Space Telescope," https://asd.gsfc.nasa.gov/firs/ (2020).

2. M. Meixner and A. Cooray, "Origins Space Telescope," https://origins.ipac.caltech.edu/.

3. C. Battersby et al. "The Origins Space Telescope," Nat. Astron. 2, 596-599 (2018).

4. D. Leisawitz et al., "The Origins Space Telescope: baseline mission concept," J. Astron. Telesc. Instrum. Syst. 7(1), 011002 (2021).

5. C. M. Bradford et al., "The Origins Survey Spectrometer (OSS): revealing the hearts of distant galaxies and forming planetary systems with far-IR spectroscopy," J. Astron. Telesc. Instrum. Syst. 7(1) (2021).

6. J. Staguhn et al., "The Origins far-infrared imager and polarimeter (FIP)," J. Astron. Telesc. Instrum. Syst. 7(1) (2021).

7. I. Sakon et al., "The Origins mid-infrared spectrometer camera (MISC) baseline and upscope," J. Astron. Telesc. Instrum. Syst. 7(1) (2021).

8. E. F. van Dishoeck, E. Herbst, and D. A. Neufeld, "Interstellar water chemistry: from laboratory to observations," Chem. Rev. 113(12), 9043-9085 (2013).

9. E. A. Bergin and E. F. van Dishoeck "Water in star- and planet-forming regions," Philos. Trans. R. Soc. A (370), 2778-2802 (2012).

10. E. A. Bergin et al., "An old disk still capable of forming a planetary system," Nature 493, 644-646 (2013).

11. S. Schiller et al., "Ultra-narrow-linewidth continuous-wave THz sources based on multiplier chains," Appl. Phys. B 95(1), 55 (2009).

12. "ALMA," http://www.almaobservatory.org/en/home/.

13. R. Guesten et al., "Performance and science opportunities with the upGREAT spectrometer onboard of SOFIA," EAS Publ. Ser. 75-76, 427-432 (2015).

14. C. Risacher et al., "The upGREAT $1.9 \mathrm{THz}$ multi-pixel high resolution spectrometer for the SOFIA observatory," Aatron. Astrophys. 595, A34 (2016).

15. T. de Graauw et al., "The Herschel-heterodyne instrument for the far-infrared (HIFI)," Astron. Astrphys. 518, L6 -L12 (2010).

16. U. Graf et al., "Terahertz heterodyne array receivers for astronomy," J. Infrared, Millimeter, Terahertz Waves 36(10), 896-921 (2015).

17. P. Goldsmith, "Sub-Millimeter heterodyne focal-plane arrays for high-resolution astronomical spectroscopy," Radio Sci. Bull. 362, 53 (2017).

18. C. Groppi et al. "Test and integration results from SuperCam: a 64-pixel array receiver for the $350 \mathrm{GHz}$ atmospheric window," Proc. SPIE 7741, 77410X (2010).

19. C. E. Groppi et al., "The kilopixel array pathfinder project (KAPPa), a 16 pixel integrated heterodyne focal plane array," Proc. SPIE 8452, 84520Y (2012).

20. U. Graf, "Heterodyne array receiver development at Kosma," in Atacama Large-Aperture Submm/mm Telescope (AtLAST), held 17-19 January, 2018 at ESO-HQ, Garching b. München, p. 9 (2018). http://www.eso.org/sci/meetings/2018/AtLAST2018.html, id.9 DOI: 10.5281/zenodo.1158854.

21. M. C. Wiedner et al., "Proposed heterodyne receiver for the Origins Space Telescope," IEEE Trans. Terahertz. Sci. Technol. 8(6), 558-571 (2019).

22. C. Groppi et al., "A broadband finline ortho-mode transducer for terahertz applications," in Proc. ISSTT, pp. 314-320 (2004). https://www.nrao.edu/meetings/isstt/proceed/2004Proceedings.pdf.

23. F. Defrance, "Instrumentation of a $2.6 \mathrm{THz}$ heterodyne receiver," $\mathrm{PhD}$, https://tel.archivesouvertes.fr/tel-01314133 (2015). 
24. K. Jacob, M. Kotiranta, and A. Murk, "Transmission and reflection characterization of polarizing beam splitters at submillimeter wavelengths," IEEE Trans. Terahertz Sci. Technol. 9(3), 272-281 (2019).

25. P. Munoz, "Waveguide heterodyne mixers at THz-frequencies-superconducting hot electron bolometers on 2 micron $\mathrm{Si}_{3} \mathrm{~N}_{4}$ membranes for GREAT and CONDOR," Ph.D. thesis, Universitaet zu Koeln (2006).

26. G. Humbert, "Optical fibers in terahertz domain," in Handbook of Optical Fibers, G. D. Peng, Ed., Springer, Singapore (2019).

27. I. Mehdi et al., "THz diode technology: status, prospects, and applications," in Proc. IEEE 105(6), 990-1007 (2017).

28. T. W. Crowe et al., "Higher power terahertz sources based on diode multipliers," in 42nd Int. Conf. Infrared, Millimeter, and Terahertz Waves, Cancun, pp. 1-1 (2017).

29. https://www.vadiodes.com/en/frequency-multipliers.

30. H.-W. Huebers et al., "High resolution terahertz spectroscopy with quantum cascade lasers," J. Infrared, Millimeter, and Terahertz Waves 34(5-6), 325-341 (2013).

31. B. Mirzaei, "Quantum cascade lasers as super terahertz local oscillators for astronomy," Ph.D. dissertation, TU Delft, Delft, The Netherlands (2018). https://repository.tudelft.nl/ islandora/object/uuid:cce70e43-8730-45e2-b645-2f8c0884b9b4?collection=research.

32. B. S. Williams et al., "Terahertz quantum-cascade metasurface VECSELs," Proc. SPIE 10123, 1012311 (2017).

33. N. van Marrewijk et al., "Frequency locking and monitoring based on bi-directional terahertz radiation of a 3rd-order distributed feedback quantum cascade laser," J. Infrared, Millimeter, and Terahertz Waves 36(12), 1210-1220 (2015).

34. Huebers, private communication.

35. H.-W. Hübers et al., "Compact and efficient 4.7-THz local oscillator with a GaAs/AlAs quantum-cascade laser," in 29th IEEE Int. Symp. Space Terahertz Technol. (2018).

36. D. Wirtz, G. Sonnabend, and R. Schieder, "THIS: a tuneable heterodyne infrared spectrometer," Spectrochim. Acta Part A: Mol. Biomol. Spectrosc. 58(11), 2457-2463 (2002).

37. A. R. Kerr et al, "The genesis of SIS mixers - the legacy of John Tucker in radioastronomy," in IEEE MTT-S Int. Microwave Symp., Phoenix, Arizona (2015).

38. A. Hedden et al., "Upgrading the SMA $600 \mathrm{GHz}$ receivers," in Proc. ISSTT, pp. 428-432 (2010).

39. A. M. Baryshev et al., "The ALMA band 9 receiver-design, construction, characterization, and first light," Astron. Astrophys. 577, A129 (2015).

40. K. Zhou et al., "Noise temperature and IF bandwidth of a $1.4 \mathrm{THz}$ superconducting HEB mixer," in Proc. URSI Asia-Pac. Radio Sci. Conf., pp. 2010-2012 (2016).

41. M. Hajenius et al., "Optimized sensitivity of $\mathrm{NbN}$ hot electron bolometer mixers by annealing," IEEE Trans. Appl. Supercond. 17(2), 399-402 (2007).

42. D. Meledin et al, "A 1.3-THz balanced waveguide HEB mixer for the APEX telescope," IEEE Trans. Microwave Theory Tech. 57(1), 89-98 (2009)

43. J. Kawamura et al., "1.9 THz 4-pixel heterodyne array receiver," in 27th Int. Symp. Space Terahertz Technol., Nanjing (2016).

44. Y. Delorme et al., "A quasi-optical NbN HEB mixer with $800 \mathrm{~K}$ DSB noise temperature at 2.5 THz," in ISSTT Proc. (2011). https://www.nrao.edu/meetings/isstt/2011.shtml.

45. J. Kawamura et al., "Development of a16 pixel monolithic $1.9 \mathrm{THz}$ waveguide superconducting hot-electron bolometer mixer," in ISSTT Conf., Cpologne (2017).

46. S. Krause et al., "Noise and IF gain bandwidth of a balanced waveguide NbN/GaN hot electron bolometer mixer operating at $1.3 \mathrm{THz}$," IEEE Trans. Terahertz Sci. Technol. 8(3), 365-371 (2018).

47. E. Novoselov and S. Cherednichenko, "Low noise terahertz $\mathrm{MgB}_{2}$ hot-electron bolometer mixers with an $11 \mathrm{GHz}$ bandwidth," Appl. Phys. Lett. 110(3), 032601 (2017)

48. J. C. Bardin, S. Montazeri, and S.-W. Chang, "Silicon germanium cryogenic low noise amplifiers," J. Phys.: Conf. Ser Conf. Series 834, 012007 (2017).

49. S. Montazeri et al., "A wide-band high-gain compact SIS receiver utilizing a $300 \mu \mathrm{W}$ SiGe IF LNA," IEEE Trans. Appl. Supercond. 27(4), 1500605 (2017). 
50. S. Montazeri et al., "Ultra-low-power cryogenic SiGe low-noise amplifiers: theory and demonstration," IEEE Trans. Microwave Theory Tech. 64(1), 178-187 (2016).

51. E. Zailer, L. Belostotski, and R. Plume, "8-GHz, 6.6-mW LC-VCO with small die area and FOM of $204 \mathrm{dBc} / \mathrm{Hz}$ at 1-MHz Offset," IEEE Microwave Wireless Comp. Lett. 26(11), 936-938 (2016).

52. A. I. Harris et al., "Cryogenic microstripline-on-Kapton microwave interconnects," Rev. Sci. Instrum. 83, 086105 (2012).

53. P. McGarey et al., "A 16-channel flex circuit for cryogenic microwave signal transmission," Proc. SPIE 9153, 91532F (2014)

54. I. Lopez-Fernandez et al., "Wideband ultra-low noise cryogenic InP IF amplifiers for the Herschel mission radiometers," Proc. SPIE 4855, 489-500 (2003).

55. Schleeh, private communication.

56. A. Tang et al., "DDFS and $\Sigma \Delta$ approaches for fractional frequency synthesis in terahertz instruments," in IEEE Trans. Terahertz Sci. Technol. 8(4), 410-417 (2018).

57. A. Tang et al., "A 177-205 GHz $249 \mathrm{~mW}$ CMOS-based integer-N frequency synthesizer module for planetary exploration," IEEE Trans. Terahertz Sci. Technol. 8(2), 251-254 (2018).

58. Y. Zhang et al., "A $2.6 \mathrm{GS} / \mathrm{s}$ spectrometer system in $65 \mathrm{~nm}$ CMOS for spaceborne telescopic sensing," in IEEE Int. Symp. Circuits and Syst., Florence, pp. 1-4 (2018).

59. Y. Kim et al., "A $1.5 \mathrm{~W} 3 \mathrm{GHz}$ back-end processor in $65 \mathrm{~m}$ CMOS for sub-millimeter-wave heterodyne receiver arrays," in Int. Symp. Space Terahertz Technol. (2018).

60. B. Klein et al., "High-resolution wide-band fast-Fourier transform spectrometers," Astron. Astrophys. 542, L3 (2012).

61. http://www.omnisys.se/product/fft-spectrometer/.

62. B. Klein et al., "A new generation of spectrometers for radio astronomy: fast Fourier transform spectrometer," Proc. SPIE 6275, 627511 (2006).

63. I. Pain et al., "SPIRE beam steering mirror: a cryogenic 2 axis mechanism for the Herschel Space Observatory," Proc. SPIE 4850, 619-627 (2003).

64. R. Huisman et al., "Cryogenic mechatronic design of the HIFI focal plane chopper," J. Mechatron. 21(8), 1259-1271 (2011).

65. S. Paalvast et al., "Development and characterization of a 2D precision cryogenic chopper for METIS,” Proc. SPIE 9151, 91510D (2014).

66. R. Huisman et al., "High-performance motion control of the METIS cold chopper mechanism," IEEE/ASME Trans. Mechatron. 21(5) (2016)

67. M. Eigenmann et al., "Conceptual phase A design of a cryogenic shutter mechanism for the SAFARI flight instrument," Proc. SPIE 8450, 84504G (2012).

68. W. Jellema, "Optical design and performance verification of Herschel-HIFI," Ph.D. University of Groningen, https://www.rug.nl/research/portal/en/publications/optical-designand-performance-verification-of-herschelhifi\%2824e22068-b3ba-45d9-b72b-503e951b193f \%29.html (2015).

69. C. Walker et al., "The stratospheric THz observatory (STO)," Proc. SPIE 7733, 77330N (2010).

70. D. W. Pesce et al., "Extremely long-baseline interferometry with the Origins Space Telescope," Astro 2020 APC White Paper, astro-ph, asXiv:1909.01408.

Martina C. Wiedner is a Centre National de la Recherche Scientifique (CNRS) researcher at Paris Observatory. She received her PhD in physics from the University of Cambridge, UK, in 1998. She held a Submillimeter Array postdoc at the Center for Astrophysics, Cambridge, USA, from 1998 to 2002, and lead a junior research group at the University of Cologne between 2003 and 2009. Her current research interests include the design, building, and testing of heterodyne receivers, and astronomical observations of star formation in the Milky Way and in other galaxies.

Edward G. Amatucci has developed everything from robotic devices to scientific instruments for space missions over the past 38 years as an engineer and currently is an instrument systems engineer as a contractor for NASA Goddard Space Flight Center. He earned a bachelor of science 
in mechanical engineering from the University of Maryland, a master of engineering in mechanical engineering, and a master of science in technical management from Johns Hopkins University.

Victor Belitsky (M'95-SM'07) received his MSc degree in electrical engineering from Moscow Telecommunication Institute, 1977, and a $\mathrm{PhD}$ in experimental physics from the Institute of Radio Engineering and Electronics, U.S.S.R. Academy of Sciences, Moscow, in 1990. He is a professor and leads the Group for Advanced Receiver Development, Department of Space, Earth, and Environment, Chalmers University, Gothenburg, Sweden. His research interests include terahertz and superconducting electronics and components, instrumentation for radio astronomy, and environmental science.

Emmanuel Caux is a CNRS senior research scientist at the Institute of Research in Astrophysics and Planetology (IRAP/OMP-UPS-CNRS-CNES, Toulouse, France). He received his $\mathrm{PhD}$ from the University of Toulouse. He was the PI of the AGLAE83 balloon-borne instrument, a co-I of the long wavelength spectrometer instrument onboard the ESA Infrared Space Observatory, and a co-PI of the HIFI instrument onboard the ESA Herschel Submillimeter Observatory. His fields of interest are the study of the ISM and star formation.

Elvire De Beck is an assistant professor at Chalmers University of Technology and currently funded by the National Swedish Space Agency. She received her MSc and PhD degrees from KULeuven, Belgium. She was a fellow at the Max Planck Institute for Radio Astronomy in Bonn, Germany, after which she took positions as APEX staff astronomer and postdoctoral researcher at Chalmers University of Technology. Her research relates mainly to evolved stars, their outflows, and mass-loss mechanisms.

Vincent Desmaris received his MSc degree in material science from the National Institute of Applied Science, Lyon, France, in 1999, and a PhD in electrical engineering from the Chalmers University of Technology, Gothenburg, Sweden, in 2006. His thesis concerned the fabrication, characterization, and modeling of AlGaN/GaN microwave transistors. Since 2006, he has been with the Group for Advanced Receiver Development, Chalmers University of Technology, where he is now a professor in microwave electronics and $\mathrm{THz}$ technology. His research interests include the terahertz receiver technology, especially the microfabrication and characterization and modeling of waveguide components and circuits, and planar cryogenic microwave devices.

Brian Ellison leads the Millimetre-wave Technology and Chilbolton Observatory Group at the Rutherford Appleton Laboratory Space Department, UK. He oversaw the UK construction contribution to the Atacama Large Millimeter/submillimeter Array and at the California Institute of Technology, USA, developed receivers for its Sub-millimeter-wave Observatory. He has led millimetre-wave instrument development programs for astronomy and Earth observation and is visiting professor at The Open University, UK, and a Fellow of the Royal Academy of Engineering.

Martin Eggens received his BSc degrees in mechanical and electrical engineering from Hogeschool Drenthe, Emmen, The Netherlands, in 1995 and 1997. Since then, he has been senior mechanical design engineer at SRON, Groningen, The Netherlands. He is mainly involved in engineering of optomechanical designs, analysis (vibration, thermal), and mechanisms of cryogenic space instrumentation like ESA/Herschel/HIFI and ground equipment. As mechanical lead engineer he was involved in the development of the SAFARI instrument for the ESA/ JAXA/SPICA mission.

Juan-Daniel Gallego is the head of the Instrumentation and Technological Development Department of the Yebes Observatory (Spain). He received his doctoral degree in physics from the Universidad Complutense (Madrid). Among other projects, he has been in charge of the development and construction of the cryogenic amplifiers of the of Herschel ESA mission and of the amplifiers for the European contribution to the ALMA. He is member of IEEE, URSI, and IAU. 
Paul F. Goldsmith is a senior research scientist at the Jet Propulsion Laboratory. He was a professor of astronomy at the University of Massachusetts, Amherst, and Cornell University. He has developed millimeter and submillimeter radiometric systems. He has carried out millimeter and submillimeter observations of molecular, atomic, and ionic fine structure lines, particularly $\mathrm{CO},[\mathrm{CI}],[\mathrm{CII}]$, [NII], and [OI] with focus to understand the structure and evolution of interstellar clouds and their relationship to star formation.

Frank Helmich is head of the Astrophysics Program at SRON Netherlands Institute for Space Research. He obtained his PhD at Leiden Observatory. He moved to SRON, where he became the principal investigator for HIFI on the Herschel Space Observatory in 2007. He has been interim executive director for the Dutch research school for astronomy NOVA. He is alternate for the Netherlands delegation to the Science Program Committee of the European Space Agency.

Fabrice Herpin is an astronomer at the Laboratoire d'Astrophysique de Bordeaux, France. He was instrument co-I of HIFI on-board Herschel Space Telescope, active member of the HIFI-Instrument Calibration Center, and Co-I of the WISH HSO Large Program. He has carried out millimeter, submillimeter, and far-IR observations of molecular lines, particularly water to understand star formation, but also various other molecules, specially $\mathrm{SiO}$ maser emission with the goal to constrain the chemical content and evolution of circumstellar envelopes of evolved stars.

Richard E. Hills, FRS, is emeritus professor of radio astronomy at the University of Cambridge, UK, having gained a BA degree there in 1967 and a PhD from UC, Berkeley, in 1973, and then held a post-doctoral position at the MPIfR, Bonn, before returning to Cambridge. He works on observational and instrumental aspects of astronomy, focusing on sub-millimetre wavelengths. He served as project scientist on JCMT and ALMA and as telescope scientist for the Planck satellite.

Michiel R. Hogerheijde is an associate professor at Leiden University and adjunct professor at the University of Amsterdam. He received his PhD from Leiden Univeristy, and held a Miller Research Fellowship at the University of California (UC), Berkeley, and a Bart J. Bok Fellowship at the University of Arizona. He has made extensive use of the Herschel Space Observatory and leads the Netherlands ALMA Regional Center node. His research focuses on the formation of stars and planets.

Leslie K. Hunt is a research director at the INAF-Arcetri Astrophysical Observatory in Firenze. After her undergraduate degree from the UC, Berkeley (USA), a master's at the University of Firenze (Italy), and her PhD from the University of Groningen (NL), she has worked at Arcetri, with a brief period at the European Space Agency for the Hubble Space Telescope in Germany. She studies mainly star-formation processes and the ISM in galaxies, and in particular the evolution of galaxies in extreme conditions.

André Laurens is a space systems expert in CNES Early Mission Studies team, and referent for Space Sciences. He received his master degree in computer science from Toulouse Science University, France. He joined CNES in the early 1980s and contributed to many space missions, working in satellite image processing, spacecraft control and command, and balloon-borne science (astrophysics and atmospheric chemistry) missions. He is conducting future mission studies in collaboration with French and international science communities.

David T. Leisawitz is a NASA study scientist for the Origins Space Telescope and is most interested scientifically in the development of habitable conditions during planet formation. He was PI on the Space Infrared Interferometric Telescope mission concept study and served as NASA Goddard study lead for the Submillimeter Probe of the Evolution of Cosmic Structure. He was a mission scientist for the Wide-field Infrared Survey Explorer and deputy project scientist for the Cosmic Background Explorer.

Dariusz C. Lis received his PhD from the University of Massachusetts, Amherst in 1989. He was a senior research associate at the California Institute of Technology and deputy director 
of the Caltech Submillimeter Observatory, professor and director of Laboratoire d'Etudes du Rayonnement et de la Matière en Astrophysique et Atmosphères at the Sorbonne University/ Paris Observatory, and is currently scientist at the Jet Propulsion Laboratory, California Institute of Technology. He is an expert in high-resolution molecular spectroscopy of the ISM and solar system small bodies.

Margaret Meixner is a director of the SOFIA Science Mission Operations center. She received her bachelor's degree in electrical engineering and math (1987) from the University of Maryland, College Park, and PhD in astronomy (1993), UC, Berkeley. Her research includes infrared instrumentation and dust evolution in galaxies. She is a member of the JWST/MIRI Science Team. She is the community co-chair of NASA's STDT for the Origins Space Telescope mission concept. Formerly, she is a JWST project scientist and distinguished astronomer at STScI. She is a fellow of the AAAS.

Gary Melnick is a senior astronomer at the Harvard-Smithsonian Center for Astrophysics specializing in infrared/submillimeter spectroscopy and astrochemistry. For more than 45 years, he has conducted airborne, balloon-borne, and space-based observations focused on the main coolants of interstellar clouds and understanding the formation, abundance, and distribution of interstellar water. He was PI of the Submillimeter Wave Astronomy Satellite (SWAS) mission, Co-I on the Herschel mission, and is the science lead for the Ices Investigation on the upcoming SPHEREx mission.

David A. Neufeld is a professor at the Johns Hopkins University in Baltimore, Maryland. He received his undergraduate degree at Clare College, Cambridge, and his $\mathrm{PhD}$ from Harvard University, and was a postdoctoral fellow at the UC Berkeley. His scientific interests include molecular astrophysics, astrochemistry, astrophysical masers, dark matter, and submillimeter and infrared astronomy.

René Plume is a professor at the University of Calgary since 2001. He obtained his BSc degree from the University of Toronto in 1989 and his PhD from the University of Texas at Austin in 1995. From 1995-2001, he was a postdoc at the Harvard-Smithsonian Center for Astrophysics working on NASA's SWAS. His background and research interests are mainly in the field of heterodyne spectroscopy of Galactic star-forming regions.

Serena Viti is a professor of astrophysics at Leiden Observatory, in the Netherlands. She received her undergraduate degree from Queen Mary University of London and her PhD from the University College London (UCL). She was a professor at UCL (where she is still affiliated to) for many years and head of the Astronomy Group until June 2020. Her research interests span a wide range of topics but are all centred around the role of molecules in space, especially in the dense gas of the ISM and star forming regions. Her recent work concentrates on the interpretation of molecular observations in extragalactic regions, and on devising novel techniques for astrochemistry involving machine learning.

Biographies of the other authors are not available. 